\title{
The Pareto-Optima of Finite-Horizon OLG MODELS
}

\author{
by
}

Charles Blackorby and Craig Brett

June 1997; revised, October, 1999

Discussion Paper No.: 99-27

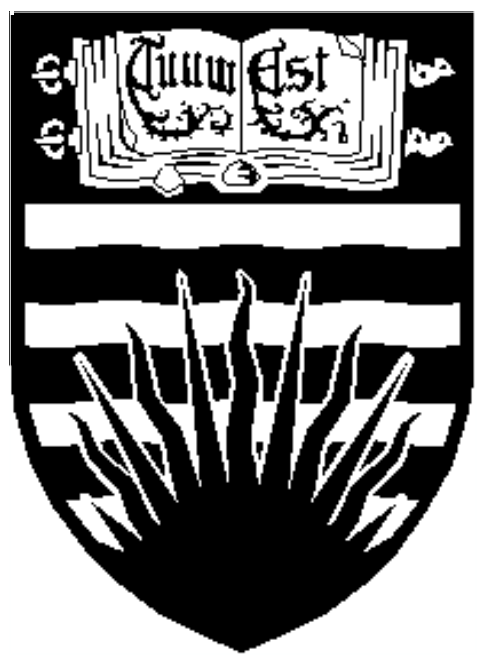

DEPARTMENT OF ECONOMICS

THE UNIVERSITY OF BRITISH COLUMBIA

VANCOUVER, CANADA V6T 1Z1

http://web.arts.ubc.ca/econ/ 


\title{
The Pareto-Optima of Finite-Horizon OLG Models
}

\author{
by \\ Charles Blackorby and Craig Brett
}

June 1997, revised, October, 1999

Charles Blackorby: Department of Economics, University of British Columbia, 997-1873 East Mall, Vancouver, BC, Canada, V6T 1Z1 and GREQAM, Centre de la Vieille Charité, 2 rue de la Charité, 13002 Marseille, France. Email: orby@econ.ubc.ca or orby@ehess.cnrs-mrs.fr

Craig Brett: Department of Economics, University of Essex, Wivenhoe Park, Colchester, Essex, United Kingdom, CO4 3SQ. Email: craig@essex.ac.uk

Acknowledgments: This research has been generously supported by the Social Science Research Council of Canada. We are indebted to Paul Beaudry for extensive comments. Blackorby is grateful for the many conversations with Francisco Gonzalez and a very productive lunch with Ivar Ekeland. 


\begin{abstract}
In a simple overlapping-generations model where the government has the power to levy commodity taxes and to implement generation-specific transfers, we show that not all the second-best optima are first-best, that is, commodity taxes and subsidies are required for a non negligible set of the efficient solutions.
\end{abstract}

Journal of Economic Literature Classification Numbers: D5, D6, D91, H2.

Keywords: overlapping generations, commodity taxes, tax-reform. 
The Pareto-Optima of Finite-Horizon OLG Models

by

Charles Blackorby and Craig Brett

\section{Introduction}

In this paper we characterize the set of Pareto-optima in a finite-horizon overlappinggenerations model where the government has the power to levy commodity taxes and to implement generation-specific transfers. This is a necessary first-step in evaluating alternative economic policies in an overlapping generations model. Are capital taxes inefficient, are taxes on savings less efficient because of the so-called double taxation, should investment in human capital be taxed in the same fashion as investment in physical capital? In order to answer such questions we need first to have some idea of the nature of the set of Pareto-optimal allocations in a model with overlapping generations. That is, we need to have a clear idea of what constitutes an efficient outcome before undertaking an analysis of the policies that would best correct some distortion.

Thus, in a finite-horizon overlapping-generations model, we give the government exactly the instruments and knowledge that would bring about a first-best allocation in a Walrasian economy and study the resulting set of Pareto-optimal allocations. We formally address the question of price normalizations; can we normalise one producer price in each period as well as one consumer price in each period? Do we need a tax on capital inputs as well as one on savings? We show that a non negligible subset of the Pareto optima in this economy are not first-best; that is, commodity taxes and subsidies are part of non negligible subset of efficient solutions. Provided that savings is positive, taxes on savings and or on capital inputs (but not both) are also required at all Paretooptima that entail some taxes. This implies that government intervention in the market 
place is essential if the economy is to achieve all of its Pareto-optima. In particular, the usual practise of assuming that consumer prices are equal to producer prices in a finite-horizon overlapping-generations model with saving may lead to an equilibrium that is Pareto-inferior to one with commodity and services taxes and generation-specific transfers.

These difficulties arise because the government has no direct mechanism for transferring income from the future to the present. This is emphasized in our model by giving only generation zero endowments. However, even if each generation had endowment in each period there would be a limit to the lump-sum transfers that the government could effectuate from the future to the present. More specifically, the transfer to generation $t-1$ cannot exceed the endowment of generation $t$ at time $t$. When this limit is reached indirect taxes and subsidies are required obtain the Pareto-optima.

There are many reasons why a government would wish to engage in such transfers. Suppose for example, that the government wants to provide a durable public good to be built in period one but wants all generations to pay for the expenditure. In fact, there always exists a government project sufficiently large so that indirect taxes and subsidies must be used to finance the public good. We do not explicitly model the government provision of public goods, but doing so is only a matter of notation.

There are several other important features of the model. In each period the government levies a hundred percent profit tax. In period one, it levies a lump-sum tax (or subsidy) on generation zero, collects specific commodity taxes (if any), and may buy a storable commodity for resale to the production sector in period two. We show that the government must balance its budget in every period. We also assume that generations one and two have positive savings (otherwise we just have a sequence of static economies), for example, generation one buys the storable good in period one and sells it to the production sector in period two. This is equivalent to buying a bond from the 
government, redeemable in period two, which the government uses to buy the storable commodity that it sells to the production sector in period two in order to redeem the bond. It is not possible for the government to spend the money in period one and to redeem the bond in period two by borrowing from generations two because the model has a finite horizon and the overall budget must be balanced. The finite horizon thus imposes structure on the model that is quite different from much of the literature. ${ }^{1}$

We use the tax reform methodology. ${ }^{2}$ Starting with an economy at an arbitrary tight equilibrium, we ask what changes in taxes, transfers, and producer prices are strictly Pareto-improving and equilibrium preserving. If there are no such changes then the economy is at a Pareto optimum. We show that these optima usually require government intervention in the market place.

The next section of the paper presents a simple finite horizon overlapping generations model with three generations. Unlike many such models in the literature, we allow for many consumption goods, in order to give some content to the issue of within period relative price changes. In Section 3 we describe the tax reform methodology and characterize strictly Pareto improving directions of policy reform. This also furnishes a description of the Pareto optima of the economy. This is followed by a section detailing the somewhat delicate issue of price normalizations. We show that producer prices and the price of capital can be normalised period by period, but that only one consumer price normalisation is admitted. The overlapping generations structure links relative prices across generations, so that independent normalisation is impossible. Only a tax on capital inputs or on saving is needed in each period, but not both. Section 5 contains

\footnotetext{
1 See, for example, Atkinson and Sandmo [1980], Balasko and Shell [1980, 1981, 1986], and Ghiglino and Shell [1998]. This is by no means the only difference. Balasko and Shell endow consumers with a linear technology - money - that permits them to move resources from on period to the other whereas Atkinson and Sandmo endow the government with such a technology that permits the carrying forward of debt.

2 See Guesnerie [1977,1995], Diewert [1978], Weymark [1979]; a good textbook treatment is in Myles [1995].
} 
a description of the geometric structure of the tax equilibria. We show that, locally, the Pareto manifold is of dimension two (one less than the number of consumers). Section 6 has concluding remarks, and Section 7 contains the many calculations needed in the text.

\section{The Model}

We consider the simplest possible overlapping generations model. ${ }^{3}$ The economy lasts for three periods: a start-up phase, a single period of the type usually examined in overlapping generations models, and a shut-down period. Given that the horizon is finite, restricting ourselves to three periods entail no loss of generality.

\subsection{Goods and Consumers}

There is a single consumer in each generation, so consumer and generation are used interchangeably. Consumers have preferences over a vector $\alpha \in \mathcal{R}^{n}$ of nonstorable goods and services; there is a storable good, $\kappa$ that is the basis for the capital stock.

An initial generation, denoted by 0 , is born old. It enters at date 1 in possession of the initial capital stock, $\kappa_{0}$. It consumes goods and services, $\alpha_{1}^{0}$, receives a generationspecific lump-sum transfer, $m_{1}$, and sells its capital stock. If any of the elements of $\alpha_{1}^{0}$ are negative these are services supplied to the market. Also alive at time 1 is a generation born young. This generation lives for two periods. During period 1, it consumes $\alpha_{1}^{1}$ and may also purchase an amount of the storable good, $\kappa_{1}^{1}$, to carry forward with it into the second period. Again, if any elements of $\alpha_{1}^{1}$ are negative, then these are services supplied to the market. In period 2 it spends its accumulated wealth and its lump-sum transfer, $m_{2}$, on the consumption of $\alpha_{2}^{1}$. Also alive in period 2 is a young generation

\footnotetext{
3 This is a simple version of the model introduced by Allais [1947], Samuelson [1958], and analyzed by Diamond [1965].
} 
which works, consumes, and saves. In period 3, the final period of our model, this generation sells its capital stock, $\kappa_{2}^{2}$, receives its lump-sum transfer, $m_{3}$, and consumes $\alpha_{3}^{3}$

The production sector is composed of an aggregate profit-maximizing firm whose technology is not assumed to be the same in every period. During periods 1 and 2, this firm can produce both $a$ and $b$ using $a$ and $k$ as inputs. In period 3 it does not produce any $b .^{4}$

\subsection{Prices}

A complete specification of a price system requires a separate price for each good, at each date in time. In addition, to allow for taxes, a set consumer prices and a set of producer prices must be specified. We express all prices in present value form. Let $p_{t}$ be the producer price vector for $a_{t}$. $\pi_{t}$ denotes the corresponding consumer price vector. $r_{t}$ is the producer price of the storable good at time $t$, while $\rho_{t}$ is its consumer price. In addition the firm buys at time $t$ the capital stock from generation $t-1$ at a price $s_{t-1}$ while generation $t-1$ receives $\sigma_{t-1}$. For the moment we make no price normalizations but show subsequently that the question of what can be normalized is a delicate issue.

\subsection{The Flows of Resources}

Besides levying specific commodity taxes, the government is assumed to tax away all pure profit $^{5}$ and it has the power to transfer income in a lump-sum to each generation. The transfer at date $t$, denoted by $m_{t}$, is paid to the old generation at that time. The planner may purchase the storable good in amounts $\kappa_{1}^{g}$ in period 1 and $\kappa_{2}^{g}$ in period

\footnotetext{
4 We use roman letters to indicate quantities produced and greek letters to indicate quantities consumed. The symbols $\alpha$ and $a$ refer to goods of identical characteristics. The same correspondence applies to $\kappa$ and $k$. An inconsistency in notation arises in that the supply of $\kappa$ is denoted $b$.

5 Alternatively, one could assume constant returns-to-scale, implying zero profits.
} 
2 to finance part of these lump sums. It has no means, however, of producing manna to bestow upon generation 0 . This makes the redistributive powers of the government less than pure lump-sum because it can engage in only those transfers implementable through capital market transactions.

Generation 0 enters the economy in possession of the capital stock, $\kappa_{0}$. It sells this capital to the firm receiving $\sigma_{0}$ per unit. It carries out its purchases with the proceeds of this sale and its lump-sum income. Given the lump-sum transfer, the budget constraint of generation 0 is 6

$$
\pi_{1}^{T} \alpha_{1}^{0} \leq \sigma_{0} \kappa_{0}+m_{1}
$$

The indirect utility function of generation 0 and its related expenditure function are given by

$$
u_{0}=V^{0}\left(\sigma_{0}, \pi_{1}, m_{1}\right) \longleftrightarrow m_{1}=E^{0}\left(u_{0}, \sigma_{0}, \pi_{1}\right) .
$$

Generation 1 uses its wage income to purchase consumption goods and services in period 1 and to buy an amount of the storable good to hold until the second period. At the beginning of the second period, it sells its capital to the firm, receiving $\sigma_{1}$ per unit. At this time, it also receives its lump-sum payment from the government. Thus, its behaviour is consistent with the joint budget constraints:

$$
\begin{aligned}
\pi_{1}^{T} \alpha_{1}^{1}+\rho_{1} \kappa_{1}^{1} & \leq 0 \\
\pi_{2}^{T} \alpha_{2}^{1} & \leq \sigma_{1} \kappa_{1}^{1}+m_{2} .
\end{aligned}
$$

Conditional on positive generation 1 saving, it faces one budget constraint that can be written as

$$
\frac{\sigma_{1}}{\rho_{1}} \pi_{1}^{T} \alpha_{1}^{1}+\pi_{2}^{T} \alpha_{2}^{1} \leq m_{2}
$$

Letting

$$
\tilde{\pi}_{1}:=\frac{\sigma_{1}}{\rho_{1}} \pi_{1}
$$

\footnotetext{
6 We use subscripts to denote the date at which a commodity is produced or consumed. When ambiguity is possible, we use superscripts to denote the birth date of the consuming agent.
} 
the indirect utility function and expenditure function - conditional on positive savings are given by

$$
u_{1}=V^{1}\left(\tilde{\pi}_{1}, \pi_{2}, m_{2}\right) \longleftrightarrow m_{2}=E^{1}\left(u_{1}, \tilde{\pi}_{1}, \pi_{2}\right) .
$$

Similarly, the value functions of generation 2-conditional on positive savings - are given by

$$
u_{2}=V^{2}\left(\tilde{\pi}_{2}, \pi_{3}, m_{3}\right) \longleftrightarrow m_{3}=E^{2}\left(u_{2}, \tilde{\pi}_{2}, \pi_{3}\right) .
$$

Each consumer maximizes lifetime utility, given the prices it faces and the lump-sum income it receives from the government. Generation 0 has a static problem. Generation 1 decides on a consumption plan for its two periods of life. Similarly for generation 2. We assume that the preferences are such that the indirect utility functions are differentially strongly quasi-convex. ${ }^{7}$

It is worth noting that the above problem imposes substantial structure on the optimal purchases of the capital stock. For example, from $(2.3), \kappa_{1}^{1}$ is homogeneous of degree zero in $\left(\pi_{1}, \rho_{1}\right)$ and $\left(\pi_{2}, \sigma_{1}, m_{2}\right)$. From $(2.4)$, it is homogeneous of degree zero in $\left(\tilde{\pi}_{1}, \pi_{2}, m_{2}\right)$. Note that the latter implies that $\kappa_{1}^{1}$ is also homogeneous of degree zero in $\left(\sigma_{1}, \rho_{1}\right)$. A similar argument holds for the purchases of $\kappa_{2}^{2}$.

Subsequently we need to know the directions of change in consumer prices and lump-sum transfers that improve the well-being of the three generations. Using Roy's theorem and the envelope conditions these can be written as

$$
\begin{gathered}
d u_{0}>0 \longleftrightarrow \kappa_{0} d \sigma_{0}-\alpha_{1}^{0 T} d \pi_{1}+d m_{1}>0 \\
d u_{1}>0 \longleftrightarrow-\frac{\sigma_{1}}{\rho_{1}} \alpha_{1}^{1 T} d \pi_{1}+\frac{\sigma_{1}}{\rho_{1}^{2}} \pi_{1}^{T} \alpha_{1}^{1} d \rho_{1}-\frac{1}{\rho_{1}} \pi_{1}^{T} \alpha_{1}^{1} d \sigma_{1}-\alpha_{2}^{1 T} d \pi_{2}+d m_{2}>0
\end{gathered}
$$

and

$$
d u_{2}>0 \longleftrightarrow-\frac{\sigma_{2}}{\rho_{2}} \alpha_{2}^{2 T} d \pi_{2}+\frac{\sigma_{2}}{\rho_{2}^{2}} \pi_{2}^{T} \alpha_{2}^{2} d \rho_{2}-\frac{1}{\rho_{2}} \pi_{2}^{T} \alpha_{2}^{2} d \sigma_{2}-\alpha_{3}^{2 T} d \pi_{3}+d m_{3}>0 .
$$

\footnotetext{
7 See Blackorby and Diewert [1979].
} 
In period 1 , the firm uses the capital it purchases from generation 0 in combination with the services supplied by generations 0 and 1 to produce a vector of (net) outputs. The profit-maximizing behaviour of the firm yields net supply functions:

$$
a_{1}=A^{1}\left(s_{0}, p_{1}, r_{1}\right), \quad b_{1}=B^{1}\left(s_{0}, p_{1}, r_{1}\right), \quad \text { and } \quad k_{1}=K^{1}\left(s_{0}, p_{1}, r_{1}\right) .
$$

In periods 2 and 3 , the firm faces essentially identical problems to the one it faced in period 1 yielding

$$
a_{2}=A^{2}\left(s_{1}, p_{2}, r_{2}\right), \quad b_{2}=B^{2}\left(s_{1}, p_{2}, r_{2}\right), \quad \text { and } \quad k_{2}=K^{2}\left(s_{1}, p_{2}, r_{2}\right)
$$

and

$$
a_{3}=A^{3}\left(s_{2}, p_{3}\right), \quad \text { and } \quad k_{3}=K^{3}\left(s_{2}, p_{3}\right) .
$$

Because the technology is not assumed to be the same in each period, this formulation is consistent with any rate of capital depreciation. We assume that the profit functions of the firm are twice continuously differentiable and strongly convex in each period. ${ }^{8}$

\subsection{Equilibrium}

Depending upon which region of the Pareto-frontier is under consideration, in periods one and two, the planner may or may not be purchasing the capital good in order to transfer resources from period $t$ to period $t+1$ for $t=1,2$. These quantities are denoted, $\kappa_{1}^{g}$ and $\kappa_{2}^{g}$ respectively. A collection of consumer and producer prices give rise

\footnotetext{
8 See Diewert, Avriel, and Zang [1981].
} 
to an equilibrium if all markets clear. The market clearing conditions are:

$$
\begin{aligned}
\kappa_{0}-k_{1} & \geq 0, \\
-\alpha_{1}^{0}-\alpha_{1}^{1}+a_{1} & \geq 0, \\
-\kappa_{1}^{1}-\kappa_{1}^{g}+b_{1} & \geq 0, \\
\kappa_{1}^{1}+\kappa_{1}^{g}-k_{2} & \geq 0, \\
-\alpha_{2}^{1}-\alpha_{2}^{2}+a_{2} & \geq 0, \\
-\kappa_{2}^{2}-\kappa_{2}^{g}+b_{2} & \geq 0, \\
\kappa_{2}^{2}+\kappa_{2}^{g}-k_{3} & \geq 0, \\
-\alpha_{3}^{2}+a_{3} & \geq 0,
\end{aligned}
$$

and the constraints on government capital purchases are given by

$$
\kappa_{1}^{g} \geq 0, \quad \text { and } \quad \kappa_{2}^{g} \geq 0
$$

It is straightforward to show that the government budget is balanced in every period. ${ }^{9}$ One is free, however, to interpret purchases of the storable good as a form of government debt.

\section{Tax Reforms}

We assume that the economy is initially in a tight equilibrium; that is, the initial vector of consumer prices, producer prices and transfer incomes is such that (2.14) all hold with equality. Depending upon the region of the Pareto-frontier being considered, either, both, or neither of (2.15) may hold with equality. This implies that there are four potentially different regimes that must be considered. For example, if both $\kappa_{1}^{g}$ and $\kappa_{2}^{g}$ are positive, then neither of two equations in (2.15) is relevant. This takes place in that region of the Pareto-frontier where the planner is moving resources away from generation zero in order to increase the demogrants to generations one and two. At

\footnotetext{
9 See Chapter 2 in Guesnerie [1995] for a general discussion of this issue; a proof is provided in the section entitled Tedious Calculations.
} 
the other extreme, both of these constraints are binding, the government would like to move resources from generation two to generations zero and one but is limited in its ability to do so by the non-negativity constraints on capital.

In addition we assume that generations one and two have positive savings. The planner may effect marginal changes in prices and generation-specific transfers. In general, the second-best outcomes that are feasible depend upon the instruments available to the government. Formally, the government has control over lump-sum transfers (using, when feasible, capital purchases) and commodity taxes while producer prices are adjusting so as to maintain equilibrium. If there are no restrictions on the use of taxes then the government can equivalently use consumer prices and the lump-sum transfers as instruments. If, however, there are restrictions on which taxes can be used these two procedures are no longer equivalent. ${ }^{10}$ Because we investigate the conditions under which some taxes may, without loss of generality, be set at zero we use producer prices, taxes and the lump-sum transfers as instruments throughout. The taxes are already defined implicitly and are given by

$$
\pi_{t}=p_{t}+\tau_{t}^{a}, \quad \sigma_{t}=s_{t}+\tau_{t}^{k}, \quad \text { and } \quad \rho_{t}=r_{t}+\tau_{t}^{b}
$$

The directions of change are given by

$$
\gamma^{T}:=\left[\gamma_{p}^{T}, \gamma_{\tau}^{T}, \gamma_{m}^{T}, \gamma_{\kappa}^{T}\right]
$$

where

$$
\begin{aligned}
\gamma_{p}^{T} & :=\left[d s_{0}, d p_{1}^{T}, d r_{1}, d s_{1}, d p_{2}^{T}, d r_{2}, d s_{2}, d p_{3}^{T}\right] ; \\
\gamma_{\tau}^{T} & :=\left[d \tau_{0}^{k}, d \tau_{1}^{a T}, d \tau_{1}^{b}, d \tau_{1}^{k}, d \tau_{2}^{a T}, d \tau_{2}^{b}, d \tau_{2}^{k}, d \tau_{3}^{a T}\right] \\
\gamma_{m}^{T} & :=\left[d m_{1}, d m_{2}, d m_{3}\right] ; \\
\gamma_{\kappa}^{T} & :=\left[d \kappa_{1}^{g}, d \kappa_{2}^{g}\right] .
\end{aligned}
$$

These vectors correspond (respectively) to changes in producer prices, taxes, lump-sum transfers, and government capital purchases.

10 See Blackorby and Brett [1998]. 


\subsection{Directions of Change}

In order to proceed we need to find those directions of change that are strictly Paretoimproving and feasible. We first collect information on those changes in consumer prices and incomes that increase utility and then those that preserve equilibrium. The relevant changes in prices and incomes in the consumer sector are given by

$$
\begin{aligned}
& P_{\pi}:= \\
& {\left[\begin{array}{cccccccc}
\kappa_{0} & -\alpha_{1}^{0 T} & 0 & 0 & 0_{n}^{T} & 0 & 0 & 0_{n}^{T} \\
0 & -\frac{\sigma_{1}}{\rho_{1}} \alpha_{1}^{1 T} & \frac{\sigma_{1}}{\rho_{1}^{2}} \pi_{1}^{T} \alpha_{1}^{1} & -\frac{1}{\rho_{1}} \pi_{1}^{T} \alpha_{1}^{1} & -\alpha_{2}^{1 T} & 0 & 0 & 0_{n}^{T} \\
0 & 0_{n}^{T} & 0 & 0 & -\frac{\sigma_{2}}{\rho_{2}} \alpha_{2}^{2 T} & \frac{\sigma_{2}}{\rho_{2}^{2}} \pi_{2}^{T} \alpha_{2}^{2} & -\frac{1}{\rho_{2}} \pi_{2}^{T} \alpha_{2}^{2} & -\alpha_{3}^{2 T}
\end{array}\right]}
\end{aligned}
$$

and

$$
P_{m}:=\left[\begin{array}{lll}
1 & 0 & 0 \\
0 & 1 & 0 \\
0 & 0 & 1
\end{array}\right]
$$

The feasibility conditions can be described in matrix form. Let $\alpha_{t}$ denote the total amount of the consumer goods demanded by all consumers alive at date $t$.

$$
E_{\pi}:=
$$$$
\left[\begin{array}{cccccccc}
0 & 0_{n}^{T} & 0 & 0 & 0_{n}^{T} & 0 & 0 & 0_{n}^{T} \\
-\nabla_{\sigma_{0}} \alpha_{1}^{0} & -\nabla_{\pi_{1}} \alpha_{1} & -\nabla_{\rho_{1}} \alpha_{1}^{1} & -\nabla_{\sigma_{1}} \alpha_{1}^{1} & -\nabla_{\pi_{2}} \alpha_{1}^{1} & 0_{n} & 0 & 0_{n \times n} \\
0 & -\nabla_{\pi_{1}} \kappa_{1}^{1} & -\nabla_{\rho_{1}} \kappa_{1}^{1} & -\nabla_{\sigma_{1}} \kappa_{1}^{1} & -\nabla_{\pi_{2}} \kappa_{1}^{1} & 0 & 0 & 0_{n}^{T} \\
0 & +\nabla_{\pi_{1}} \kappa_{1}^{1} & +\nabla_{\rho_{1}} \kappa_{1}^{1} & +\nabla_{\sigma_{1}} \kappa_{1}^{1} & +\nabla_{\pi_{2}} \kappa_{1}^{1} & 0 & 0 & 0_{n}^{T} \\
0 & -\nabla_{\pi_{1}} \alpha_{2}^{1} & -\nabla_{\rho_{1}} \alpha_{2}^{1} & -\nabla_{\sigma_{1}} \alpha_{2}^{1} & -\nabla_{\pi_{2}} \alpha_{2} & -\nabla_{\rho_{2}} \alpha_{2}^{2} & -\nabla_{\sigma_{2}} \alpha_{2}^{2} & -\nabla_{\pi_{3}} \alpha_{2}^{2} \\
0 & 0_{n}^{T} & 0 & 0 & -\nabla_{\pi_{2}} \kappa_{2}^{2} & -\nabla_{\rho_{2}} \kappa_{2}^{2} & -\nabla_{\sigma_{2}} \kappa_{2}^{2} & -\nabla_{\pi_{3}} \kappa_{2}^{2} \\
0 & 0_{n}^{T} & 0 & 0 & +\nabla_{\pi_{2}} \kappa_{2}^{2} & +\nabla_{\rho_{2}} \kappa_{2}^{2} & +\nabla_{\sigma_{2}} \kappa_{2}^{2} & +\nabla_{\pi_{3}} \kappa_{2}^{2} \\
0_{n} & 0_{n \times n} & 0_{n} & 0_{n} & -\nabla_{\pi_{2}} \alpha_{3}^{2} & -\nabla_{\rho_{2}} \alpha_{3}^{2} & -\nabla_{\sigma_{2}} \alpha_{3}^{2} & -\nabla_{\pi_{3}} \alpha_{3}^{2}
\end{array}\right]
$$

$$
E_{m}:=\left[\begin{array}{ccc}
0 & 0 & 0 \\
-\nabla_{m_{1}} \alpha_{1}^{0} & -\nabla_{m_{2}} \alpha_{1}^{1} & 0 \\
0 & -\nabla_{m_{2}} \kappa_{1}^{1} & 0 \\
0 & \nabla_{m_{2}} \kappa_{1}^{1} & 0 \\
0 & -\nabla_{m_{2}} \alpha_{2}^{1} & -\nabla_{m_{3}} \alpha_{2}^{2} \\
0 & 0 & -\nabla_{m_{3}} \kappa_{2}^{2} \\
0 & 0 & \nabla_{m_{3}} \kappa_{2}^{2} \\
0 & 0 & -\nabla_{m_{3}} \alpha_{3}^{2}
\end{array}\right]
$$




$$
\begin{aligned}
& E_{p}:= \\
& {\left[\begin{array}{cccccccc}
-\nabla_{s_{0}} k_{1} & -\nabla_{p_{1}} k_{1} & -\nabla_{r_{1}} k_{1} & 0 & 0_{n}^{T} & 0 & 0 & 0_{n}^{T} \\
\nabla_{s_{0}} a_{1} & \nabla_{p_{1}} a_{1} & \nabla_{r_{1}} a_{1} & 0_{n} & 0_{n \times n} & 0_{n} & 0_{n} & 0_{n \times n} \\
\nabla_{s_{0}} b_{1} & \nabla_{p_{1}} b_{1} & \nabla_{r_{1}} b_{1} & 0 & 0_{n}^{T} & 0 & 0 & 0_{n}^{T} \\
0 & 0_{n}^{T} & 0 & -\nabla_{s_{1}} k_{2} & -\nabla_{p_{2}} k_{2} & -\nabla_{r_{2}} k_{2} & 0 & 0_{n}^{T} \\
0_{n} & 0_{n \times n} & 0_{n} & \nabla_{s_{1}} a_{2} & \nabla_{p_{2}} a_{2} & \nabla_{r_{2}} a_{2} & 0 & 0_{n}^{T} \\
0 & 0_{n}^{T} & 0 & \nabla_{s_{1}} b_{2} & \nabla_{p_{2}} b_{2} & \nabla_{r_{2}} b_{2} & 0 & 0_{n}^{T} \\
0 & 0_{n}^{T} & 0 & 0 & 0_{n}^{T} & 0 & -\nabla_{s_{2}} k_{3} & -\nabla_{p_{3}} k_{3} \\
0_{n} & 0_{n \times n} & 0_{n} & 0_{n} & 0_{n \times n} & 0_{n} & \nabla_{s_{2}} a_{3} & \nabla_{p_{3}} a_{3}
\end{array}\right] ;}
\end{aligned}
$$

and

$$
E_{\kappa}:=\left[\begin{array}{cc}
0 & 0 \\
0 & 0 \\
-1 & 0 \\
1 & 0 \\
0 & 0 \\
0 & -1 \\
0 & 1 \\
0 & 0
\end{array}\right]
$$

In addition to the above we have the nonnegativity constraint on the governments purchases of capital,

$$
d k_{1}^{g}+k_{1}^{g} \geq 0 \quad \text { and } \quad d k_{2}^{g}+k_{2}^{g} \geq 0 .
$$

\subsection{Strictly Pareto-Improving Equilibrium Preserving Directions of Change}

A set of changes is strictly Pareto-improving if and only if

$$
P_{\pi} \gamma_{p}+P_{\pi} \gamma_{\tau}+P_{m} \gamma_{m}+\mathbf{0} \gamma_{\kappa} \gg 0
$$

where $\mathbf{0}$ is an appropriately dimensioned matrix of zeros. A direction is equilibriumpreserving if and only if

$$
\left[E_{\pi}+E_{p}\right] \gamma_{p}+E_{\pi} \gamma_{\tau}+E_{m} \gamma_{m}+E_{\kappa} \gamma_{\kappa} \geq 0
$$

In addition the capital constraints on government must be satisfied, (3.10). There are strict Pareto-improving changes that are simultaneously equilibrium-preserving if 
and only (3.11), (3.12), and (3.10) have a solution. Together these constitute a non homogeneous system which we convert to the following homogeneous system. ${ }^{11}$

$$
\left[\begin{array}{ccccc}
P_{\pi} & P_{\pi} & P_{m} & 0_{n \times 2} & 0_{n} \\
0_{n}^{T} & 0_{n}^{T} & 0_{3}^{T} & 0_{2}^{T} & 1
\end{array}\right]\left[\begin{array}{c}
\gamma \\
\gamma_{\eta}
\end{array}\right] \gg 0
$$

and

$$
\left[\begin{array}{ccccc}
E_{\pi}+E_{p} & E_{\pi} & E_{m} & E_{\kappa} & 0 \\
0_{2 \times n} & 0_{2 \times n} & 0_{2 \times 3} & I_{2 \times 2} & \kappa^{g}
\end{array}\right]\left[\begin{array}{c}
\gamma \\
\gamma_{\eta}
\end{array}\right] \geq 0
$$

where $\gamma_{\eta}$ is the dummy variable used to convert the non homogeneous system to a homogeneous one. If there is no solution we are at a second-best optimum. Using Motzkin's Theorem ${ }^{12}$ the economy is at a second-best optimum if and only if

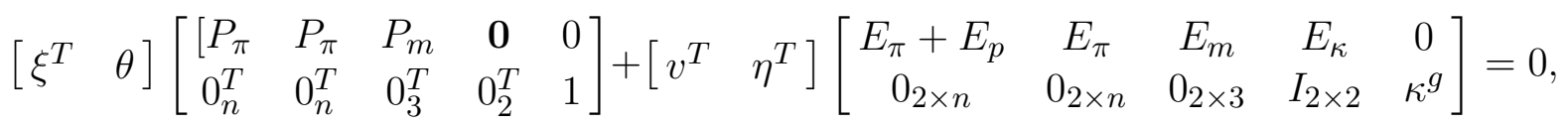

where $0 \neq\left[\xi^{T}, \theta\right] \geq 0^{T}$ and $\left[v^{T}, \eta^{T}\right] \geq 0^{T}$.

Before analyzing the structure of the Pareto-optimal set and the implied taxes and subsidies, we examine the question of price-normalizations. That is, how many producer and consumer prices are actually redundant?

\section{Normalizations}

In this section we investigate the legitimacy of normalizing prices. We do this formally by adding constraints to the original system of equations preventing some prices from changing. If the multiplier on this constraint can be shown to be zero, then this normalization places no restrictions on the optimal solution and is permissible. If however, this multiplier cannot be shown to be zero, then such a normalization is inconsistent with an efficient equilibrium. The results of this exercise are summarized at the end of this section. It is important to note that if one normalizes more prices than are

11 See the second subsection of Tedious Calculations for this argument.

12 See Mangasarian [1969, pp. 28-29] for a statement and proof of this result. 
admitted by the above procedure, then the resulting set of equilibria are not necessarily Pareto-optimal.

\subsection{Producer Price Normalizations}

First we show that at most one producer price can be normalized in each period. To set

$$
s_{0}=1, \quad s_{1}=1, \quad \text { and } \quad s_{2}=1
$$

and to look for the Pareto-improving directions that are equilibrium-preserving and satisfy (4.1) define

$$
\mathcal{I}=\left[\begin{array}{llllllll}
1 & 0_{n}^{T} & 0 & 0 & 0_{n}^{T} & 0 & 0 & 0_{n}^{T} \\
0 & 0_{n}^{T} & 0 & 1 & 0_{n}^{T} & 0 & 0 & 0_{n}^{T} \\
0 & 0_{n}^{T} & 0 & 0 & 0_{n}^{T} & 0 & 1 & 0_{n}^{T}
\end{array}\right]
$$

(4.1) is satisfied if and only if

$$
\mathcal{I} \gamma_{p}+\mathbf{0} \gamma_{\tau}+\mathbf{0} \gamma_{m}+\mathbf{0} \gamma_{\kappa}+\mathbf{0} \gamma_{\eta}=0
$$

where $\mathbf{0}$ is an appropriately dimensioned block of zeroes.

There are strict Pareto-improving changes that are simultaneously equilibriumpreserving with three producer price normalizations if and only if $(3.13),(3.14)$, and(4.3) have a solution. If there is no such solution we are at a second-best optimum. Using Motzkin's Theorem the economy is at a second-best optimum if and only if

$$
\begin{array}{r}
{\left[\begin{array}{ll}
\xi^{T} & \theta
\end{array}\right]\left[\begin{array}{ccccc}
{\left[P_{\pi}\right.} & P_{\pi} & P_{m} & \mathbf{0} & 0 \\
0_{n}^{T} & 0_{n}^{T} & 0_{3}^{T} & 0_{2}^{T} & 1
\end{array}\right]+\left[\begin{array}{ll}
v^{T} & \eta^{T}
\end{array}\right]\left[\begin{array}{ccccc}
E_{\pi}+E_{p} & E_{\pi} & E_{m} & E_{\kappa} & 0 \\
0_{2 \times n} & 0_{2 \times n} & 0_{2 \times 3} & I_{2 \times 2} & \kappa^{g}
\end{array}\right]} \\
\\
+w^{T}\left[\begin{array}{lllll}
\mathcal{I} & \mathbf{0} & \mathbf{0} & \mathbf{0} & \mathbf{0}
\end{array}\right]=0,
\end{array}
$$

or, expanding

$$
\begin{gathered}
\xi^{T} P_{\pi}+v^{T}\left[E_{\pi}+E_{p}\right]+w^{T} \mathcal{I}=0, \\
\xi^{T} P_{\pi}+v^{T} E_{\pi}=0
\end{gathered}
$$




$$
\begin{gathered}
\xi^{T} P_{m}+v^{T} E_{m}=0 . \\
v^{T} E_{\kappa}+\eta^{T}=0,
\end{gathered}
$$

and

$$
\theta+\eta^{T} k^{g}=0
$$

Subtracting (4.6) from (4.5) yields

$$
v^{T} E_{p}+w^{T} \mathcal{I}=0
$$

or, using (3.8),

$$
\begin{gathered}
v^{1 T}\left[\begin{array}{ccc}
-\nabla_{s_{0}} k_{1} & -\nabla_{p_{1}} k_{1} & -\nabla_{r_{1}} k_{1} \\
\nabla_{s_{0}} a_{1} & \nabla_{p_{1}} a_{1} & \nabla_{r_{1}} a_{1} \\
\nabla_{s_{0}} b_{1} & \nabla_{p_{1}} b_{1} & \nabla_{r_{1}} b_{1}
\end{array}\right]+\left(w_{1}, 0_{n}^{T}, 0\right)=0, \\
v^{2 T}\left[\begin{array}{ccc}
-\nabla_{s_{1}} k_{2} & -\nabla_{p_{2}} k_{2} & -\nabla_{r_{2}} k_{2} \\
\nabla_{s_{1}} a_{2} & \nabla_{p_{2}} a_{2} & \nabla_{r_{2}} a_{2} \\
\nabla_{s_{1}} b_{2} & \nabla_{p_{2}} b_{2} & \nabla_{r_{2}} b_{2}
\end{array}\right]+\left(w_{2}, 0_{n}^{T}, 0\right)=0,
\end{gathered}
$$

and

$$
v^{3 T}\left[\begin{array}{cc}
-\nabla_{s_{2}} k_{3} & -\nabla_{p_{3}} k_{3} \\
\nabla_{s_{2}} a_{3} & \nabla_{p_{3}} a_{3}
\end{array}\right]+\left(w_{3}, 0_{n}^{T}\right)=0
$$

where $v^{T}=\left(v^{1 T}, v^{2 T}, v^{3 T}\right)$ is defined implicitly by the above; $v^{1 T}$ and $v^{2 T}$ are $n+2$ tuples, and $v^{3 T}$ is a $n+1$ tuple. Post-multiply (4.11) by the producer price vector to obtain

$$
w_{1} s_{0}=0, \quad w_{2} s_{1}=0 \quad \text { and } \quad w_{3} s_{2}=0
$$

Because prices are positive, this implies that $w=0$ and hence that the constraints embodying the normalizations do not bind at the second-best optimum; that is, (4.1) places no restrictions on the optimum. It is easy to see that if another producer price were normalized then some of these constraints would be strictly binding. 


\subsection{Producer and Capital Input (or Savings) Price Normalizations}

Before proceeding to a formal analysis first note that intuition suggests that several prices can be normalised without loss of generality. The budget constraint of generation zero is given by

$$
\pi_{1} \alpha_{1}^{0} \leq\left(s_{0}+\tau_{0}^{k}\right) \kappa_{0}+m_{1}
$$

Clearly the tax on the fixed amount of the capital stock, $\tau_{0}^{k}$, is a perfect substitute for the lump-sum transfer, $m_{1}$, and is redundant in a Pareto-optimum. Supposing that savings is positive, the budget constraint of generation one is given by

$$
\left[\frac{s_{1}+\tau_{1}^{k}}{r_{1}+\tau_{1}^{b}}\right] \pi_{1} \alpha_{1}^{1}+\pi_{2} \alpha_{2}^{1} \leq m_{2} .
$$

constraint of generation two is given by

$$
\left[\frac{s_{2}+\tau_{2}^{k}}{r_{2}+\tau_{2}^{b}}\right] \pi_{2} \alpha_{2}^{2}+\pi_{3} \alpha_{3}^{2} \leq m_{3} .
$$

show formally that these suggestions are correct.

Suppose now, that in addition to (4.1) we constrain the taxes on the inputs purchases to be zero, that is,

$$
\tau_{0}^{k}=0, \quad \tau_{1}^{k}=0, \quad \text { and } \quad \tau_{2}^{k}=0
$$

A direction is strictly Pareto-improving and equilibrium-preserving when the price normalizations (4.1) and (4.16) are satisfied if and only if (3.13), (3.14), and

$$
\left[\begin{array}{l}
\mathcal{I} \\
\mathbf{0}
\end{array}\right] \gamma_{p}+\left[\begin{array}{l}
\mathbf{0} \\
\mathcal{I}
\end{array}\right] \gamma_{\tau}+\left[\begin{array}{l}
\mathbf{0} \\
\mathbf{0}
\end{array}\right] \gamma_{m}\left[\begin{array}{l}
\mathbf{0} \\
\mathbf{0}
\end{array}\right] \gamma_{\kappa}+\left[\begin{array}{l}
\mathbf{0} \\
\mathbf{0}
\end{array}\right] \gamma_{\eta}=0
$$

are satisfied. If there is no such solution we are at a second-best optimum. Using Motzkin's Theorem the economy is at a second-best optimum if and only if

$$
\begin{aligned}
& {\left[\begin{array}{ll}
\xi^{T} & \theta
\end{array}\right]\left[\begin{array}{ccccc}
{\left[P_{\pi}\right.} & P_{\pi} & P_{m} & \mathbf{0} & 0 \\
0_{n}^{T} & 0_{n}^{T} & 0_{3}^{T} & 0_{2}^{T} & 1
\end{array}\right]+\left[\begin{array}{ll}
v^{T} & \eta^{T}
\end{array}\right]\left[\begin{array}{ccccc}
E_{\pi}+E_{p} & E_{\pi} & E_{m} & E_{\kappa} & 0 \\
0_{2 \times n} & 0_{2 \times n} & 0_{2 \times 3} & I_{2 \times 2} & \kappa^{g}
\end{array}\right]} \\
& +\left(w^{T}, z^{T}\right)\left[\begin{array}{lllll}
\mathcal{I} & \mathbf{0} & \mathbf{0} & \mathbf{0} & \mathbf{0} \\
\mathbf{0} & \mathcal{I} & \mathbf{0} & \mathbf{0} & \mathbf{0}
\end{array}\right]=0
\end{aligned}
$$


or, expanding

$$
\begin{gathered}
\xi^{T} P_{\pi}+v^{T}\left[E_{\pi}+E_{p}\right]+w^{T} \mathcal{I}=0, \\
\xi^{T} P_{\pi}+v^{T} E_{\pi}+z^{T} \mathcal{I}=0, \\
\xi^{T} P_{m}+v^{T} E_{m}=0, \\
v^{T} E_{\kappa}+\eta^{T}=0,
\end{gathered}
$$

and

$$
\theta+\eta^{T} \kappa^{g}=0
$$

This means that we cannot simply proceed as above, subtracting (4.19) from (4.18). If we are to show that some consumer prices can be normalized without loss of generality then this now has to come from (4.20). Only when the shadow value of the constraints on consumer prices are shown to be identically zero can we proceed as above. Let

$$
v^{T}=\left(v_{1}, v_{2}^{T}, v_{3}, v_{4}, v_{5}^{T}, v_{6}, v_{7}, v_{8}^{T}\right)
$$

For this to be consistent with the above,

$$
v^{1 T}=\left(v_{1}, v_{2}^{T}, v_{3}\right), v^{2 T}=\left(v_{4}, v_{5}^{T}, v_{6}\right), \text { and } v^{3 T}=\left(v_{7}, v_{8}^{T}\right) .
$$

Expanding (4.21) yields

$$
\begin{gathered}
\xi_{1}=v_{2}^{T} \nabla_{m_{1}} \alpha_{1}^{0}, \\
\xi_{2}=v_{2}^{T} \nabla_{m_{2}} \alpha_{1}^{1}+\left(v_{3}-v_{4}\right) \nabla_{m_{2}} \kappa_{1}^{1}+v_{5}^{T} \nabla_{m_{2}} \alpha_{2}^{1},
\end{gathered}
$$

and

$$
\xi_{3}=v_{5}^{T} \nabla_{m_{3}} \alpha_{2}^{2}+\left(v_{6}-v_{7}\right) \nabla_{m_{3}} \kappa_{2}^{2}+v_{8}^{T} \nabla_{m_{3}} \alpha_{3}^{2}
$$

Expanding (4.20), using (4.26)-(4.28), and the Slutsky equation repeatedly yields ${ }^{13}$

$$
-\xi_{1} \kappa_{0}=-v_{2}^{T} \nabla_{\sigma_{0}} \alpha_{1}^{0}+z_{1}
$$

13 See the normalisation subsection in Tedious Calculations. 


$$
\begin{aligned}
& {\left[\begin{array}{lll}
v_{2}^{T} & v_{5}^{T} & v_{8}^{T}
\end{array}\right]\left[\begin{array}{ccc}
E_{\pi_{1} \pi_{1}}^{0}+\frac{\sigma_{1}}{\rho_{1}} E_{\tilde{\pi}_{1} \tilde{\pi}_{1}}^{1} & E_{\tilde{\pi}_{1} \pi_{2}}^{1} & 0 \\
\frac{\sigma_{1}}{\rho_{1}} E_{\pi_{2} \tilde{\pi}_{1}}^{1} & E_{\pi_{2} \pi_{2}}^{1}+\frac{\sigma_{2}}{\rho_{2}} E_{\tilde{\pi}_{2} \tilde{\pi}_{2}}^{2} & E_{\tilde{\pi}_{2} \pi_{3}}^{2} \\
0 & \frac{\sigma_{2}}{\rho_{2}} E_{\pi_{3} \tilde{\pi}_{2}}^{2} & E_{\pi_{3} \pi_{3}}^{2}
\end{array}\right]=} \\
& {\left[\begin{array}{c}
\left(v_{4}-v_{3}\right)\left(\nabla_{\pi_{1}} \kappa_{1}^{1}+\nabla_{m_{2}} \kappa_{1}^{1} \frac{\sigma_{1}}{\rho_{1}} \alpha_{1}^{1 T}\right) \\
\left(v_{4}-v_{3}\right)\left(\nabla_{\pi_{2}} \kappa_{1}^{1}+\nabla_{m_{2}} \kappa_{1}^{1} \alpha_{2}^{1 T}\right)+\left(v_{7}-v_{6}\right)\left(\nabla_{\pi_{2}} \kappa_{2}^{2}+\nabla_{m_{3}} \kappa_{2}^{2} \frac{\sigma_{2}}{\rho_{2}} \alpha_{2}^{2 T}\right) \\
\left(v_{7}-v_{6}\right)\left(\nabla_{\pi_{3}} \kappa_{2}^{2}+\nabla_{m_{3}} \kappa_{2}^{2} \alpha_{3}^{2 T}\right)
\end{array}\right]} \\
& v_{2}^{T} \frac{\sigma_{1}}{\rho_{1}^{2}} E_{\tilde{\pi}_{1} \tilde{\pi}_{1}}^{1} \pi_{1}+v_{5}^{T} \frac{\sigma_{1}}{\rho_{1}^{2}} E_{\pi_{2} \tilde{\pi}_{1}}^{1} \pi_{1}= \\
& \left(v_{4}-v_{3}\right)\left(\nabla_{m_{2}} \kappa_{1}^{1} \frac{\sigma_{1}}{\rho_{1}^{2}} \pi_{1}^{T} \alpha_{1}^{1}-\nabla_{\rho_{1}} \kappa_{1}^{1}\right) \\
& v_{2}^{T} \frac{1}{\rho_{1}} E_{\tilde{\pi}_{1} \tilde{\pi}_{1}}^{1} \pi_{1}+v_{5}^{T} \frac{1}{\rho_{1}} E_{\pi_{2} \tilde{\pi}_{1}}^{1} \pi_{1}= \\
& \left(v_{4}-v_{3}\right)\left(\nabla_{\sigma_{1}} \kappa_{1}^{1}+\nabla_{m_{2}} \kappa_{1}^{1} \frac{1}{\rho_{1}} \pi_{1}^{T} \alpha_{1}^{1}\right)+z_{2}, \\
& v_{5}^{T} \frac{\sigma_{2}}{\rho_{2}^{2}} E_{\tilde{\pi}_{2} \tilde{\pi}_{2}}^{2} \pi_{2}+v_{8}^{T} \frac{\sigma_{2}}{\rho_{2}^{2}} E_{\pi_{3} \tilde{\pi}_{2}}^{2} \pi_{2}= \\
& \left(v_{7}-v_{6}\right)\left(\nabla_{m_{3}} \kappa_{2}^{2} \frac{\sigma_{2}}{\rho_{2}^{2}} \alpha_{2}^{2 T} \pi_{2}-\nabla_{\rho_{2}} \kappa_{2}^{2}\right),
\end{aligned}
$$

and

$$
\begin{array}{r}
v_{5}^{T} \frac{1}{\rho_{2}} E_{\tilde{\pi}_{2} \tilde{\pi}_{2}} \pi_{2}+v_{8}^{T} \frac{1}{\rho_{2}} E_{\pi_{3} \tilde{\pi}_{2}}^{2} \pi_{2}= \\
\left(v_{7}-v_{6}\right)\left(\nabla_{\sigma_{2}} \kappa_{2}^{2}+\nabla_{m_{3}} \kappa_{2}^{2} \frac{1}{\rho_{2}} \alpha_{2}^{2 T} \pi_{2}\right)+z_{3},
\end{array}
$$

First note that $\tau_{0}^{k}$ can be set equal to zero without any loss of generality. Multiply (4.26) by $\kappa_{0}$ and add it to (4.29) to obtain

$$
z_{1}=0
$$

This means that the input taxes on capital in period one can be set equal to zero without loss of generality. This is a consequence of the start-up features of the model. The income of generation zero is given by $\kappa_{0}\left(s_{0}+t_{0}^{k}\right)+m_{1}$ so that taxing the fixed capital stock is the same as reducing the lump-sum transfer. This tax is therefore redundant and confirms the above intuition. 
Multiply (4.31) by $\rho_{1},(4.32)$ by $\sigma_{1}$ and subtract to obtain

$$
\left(v_{4}-v_{3}\right)\left(\rho_{1} \nabla_{\rho_{1}} \kappa_{1}^{1}+\sigma_{1} \nabla_{\sigma_{1}} \kappa_{1}^{1}\right)+z_{2} \sigma_{1}=0
$$

similarly from (4.33) and (4.34) obtain

$$
\left(v_{7}-v_{6}\right)\left(\rho_{2} \nabla_{\rho_{2}} \kappa_{2}^{2}+\sigma_{2} \nabla_{\sigma_{2}} \kappa_{2}^{2}\right)+z_{3} \sigma_{2}=0
$$

Because $\kappa_{t}$ is homogeneous of degree zero in $\left(\rho_{t}, \sigma_{t}\right),(4.36)$ and $(4.37)$ imply that

$$
z_{2} \sigma_{1}=0 \quad \text { and } \quad z_{3} \sigma_{2}=0
$$

which in turn implies that $z_{2}=0$ and $z_{3}=0$, the normalizations of the input prices are not binding. It is clear from the above argument that instead of setting $\tau_{1}^{k}=0$, we could have set $\tau_{1}^{b}=0$, but not both. Similarly we could have chosen to normalise at $\tau_{2}^{b}=0$ instead of setting $\tau_{2}^{k}=0$, but again, not both. This too confirms our above stated intuition. Now subtract (4.19) from (4.18) and as in the previous subsection and we find still that one producer price can be normalized in each period.

\subsection{Consumer Prices Too}

Finally we address the question of how many consumer prices can be normalised. In order to make the argument clear we normalise one consumer price in period one and one consumer price in period two and show that, in conjunction with what has been assumed already, this is not consistent with the achievement of a Pareto-optimum; hence only one consumer price can be normalised.

Suppose that in addition to (4.1) and (4.16) we set the first component of $\tau_{1}^{a}$ equal to zero and the first component of $\tau_{2}^{a}$ equal to zero. Let

$$
\tilde{\mathcal{I}}=\left[\begin{array}{cccccccc}
1 & 0_{n}^{T} & 0 & 0 & 0_{n}^{T} & 0 & 0 & 0_{n}^{T} \\
0 & 1,0_{n-1}^{T} & 0 & 0 & 0_{n}^{T} & 0 & 0 & 0_{n}^{T} \\
0 & 0_{n}^{T} & 0 & 1 & 0_{n}^{T} & 0 & 0 & 0_{n}^{T} \\
0 & 0_{n}^{T} & 0 & 0 & 1,0_{n-1}^{T} & 0 & 0 & 0_{n}^{T} \\
0 & 0_{n}^{T} & 0 & 0 & 0_{n}^{T} & 0 & 1 & 0_{n}^{T}
\end{array}\right] .
$$


These normalizations are imposed by

$$
\left[\begin{array}{l}
\mathcal{I} \\
\mathbf{0}
\end{array}\right] \gamma_{p}+\left[\begin{array}{l}
\mathbf{0} \\
\tilde{\mathcal{I}}
\end{array}\right] \gamma_{\tau}+\left[\begin{array}{l}
\mathbf{0} \\
\mathbf{0}
\end{array}\right] \gamma_{m}+\left[\begin{array}{l}
\mathbf{0} \\
\mathbf{0}
\end{array}\right] \gamma_{\kappa}+\left[\begin{array}{l}
\mathbf{0} \\
\mathbf{0}
\end{array}\right] \gamma_{\eta}=0
$$

There are strict Pareto-improving changes that are simultaneously equilibriumpreserving with three producer price normalizations and three capital inputs price normalizations and two consumer price normalizations if and only (3.13), (3.14), and (4.40) have a solution. If there is no such solution we are at a second-best optimum. Using Motzkin's Theorem the economy is at a second-best optimum if and only if

$$
\begin{aligned}
& {\left[\begin{array}{ll}
\xi^{T} & \theta
\end{array}\right]\left[\begin{array}{ccccc}
{\left[P_{\pi}\right.} & P_{\pi} & P_{m} & \mathbf{0} & 0 \\
0_{n}^{T} & 0_{n}^{T} & 0_{3}^{T} & 0_{2}^{T} & 1
\end{array}\right]+\left[\begin{array}{cc}
v^{T} & \eta^{T}
\end{array}\right]\left[\begin{array}{ccccc}
E_{\pi}+E_{p} & E_{\pi} & E_{m} & E_{\kappa} & 0 \\
0_{2 \times n} & 0_{2 \times n} & 0_{2 \times 3} & I_{2 \times 2} & \kappa^{g}
\end{array}\right]} \\
& +\left(w^{T}, z^{T}\right)\left[\begin{array}{ccccc}
\mathcal{I} & \mathbf{0} & \mathbf{0} & \mathbf{0} & \mathbf{0} \\
\mathbf{0} & \tilde{\mathcal{I}} & \mathbf{0} & \mathbf{0} & \mathbf{0}
\end{array}\right]=0
\end{aligned}
$$

Expanding (4.41) yields

$$
\begin{gathered}
\xi_{1}=v_{2}^{T} \nabla_{m_{1}} \alpha_{1}^{0}, \\
\xi_{2}=v_{2}^{T} \nabla_{m_{2}} \alpha_{1}^{1}+\left(v_{3}-v_{4}\right) \nabla_{m_{2}} \kappa_{1}^{1}+v_{5}^{T} \nabla_{m_{2}} \alpha_{2}^{1},
\end{gathered}
$$

and

$$
\xi_{3}=v_{5}^{T} \nabla_{m_{3}} \alpha_{2}^{2}+\left(v_{6}-v_{7}\right) \nabla_{m_{3}} \kappa_{2}^{2}+v_{8}^{T} \nabla_{m_{3}} \alpha_{3}^{2}
$$

Expanding (4.41), using (4.42)-(4.44), and the Slutsky equation repeatedly yields ${ }^{14}$

$$
\begin{gathered}
-\xi_{1} \kappa_{0}=-v_{2}^{T} \nabla_{\sigma_{0}} \alpha_{1}^{0}+\tilde{z}_{1} \\
{\left[\begin{array}{lll}
v_{2}^{T} & v_{5}^{T} & v_{8}^{T}
\end{array}\right]\left[\begin{array}{ccc}
E_{\pi_{1} \pi_{1}}^{0}+\frac{\sigma_{1}}{\rho_{1}} E_{\tilde{\pi}_{1} \tilde{\pi}_{1}}^{1} & E_{\tilde{\pi}_{1} \pi_{2}}^{1} & 0 \\
\frac{\sigma_{1}}{\rho_{1}} E_{\pi_{2} \tilde{\pi}_{1}}^{1} & E_{\pi_{2} \pi_{2}}^{1}+\frac{\sigma_{2}}{\rho_{2}} E_{\tilde{\pi}_{2} \tilde{\pi}_{2}}^{2} & E_{\tilde{\pi}_{2} \pi_{3}}^{2} \\
0 & \frac{\sigma_{2}}{\rho_{2}} E_{\pi_{3} \tilde{\pi}_{2}}^{2} & E_{\pi_{3} \pi_{3}}^{2}
\end{array}\right]=} \\
{\left[\begin{array}{c}
\left(v_{4}-v_{3}\right)\left(\nabla_{\pi_{1}} \kappa_{1}^{1}+\nabla_{m_{2}} \kappa_{1}^{1} \frac{\sigma_{1}}{\rho_{1}} \alpha_{1}^{1 T}\right)+\left(\tilde{z}_{2}, 0_{n-1}^{T}\right) \\
\left(v_{4}-v_{3}\right)\left(\nabla_{\pi_{2}} \kappa_{1}^{1}+\nabla_{m_{2}} \kappa_{1}^{1} \alpha_{2}^{1 T}\right)+\left(v_{7}-v_{6}\right)\left(\nabla_{\pi_{2}} \kappa_{2}^{2}+\nabla_{m_{3}} \kappa_{2}^{2} \frac{\sigma_{2}}{\rho_{2}} \alpha_{2}^{2 T}\right)+\left(\tilde{z}_{4}, 0_{n-1}^{T}\right) \\
\left(v_{7}-v_{6}\right)\left(\nabla_{\pi_{3}} \kappa_{2}^{2}+\nabla_{m_{3}} \kappa_{2}^{2} \alpha_{3}^{2 T}\right)
\end{array}\right]}
\end{gathered}
$$

14 See the normalisation subsection in Tedious Calculations. 


$$
\begin{gathered}
v_{2}^{T} \frac{\sigma_{1}}{\rho_{1}^{2}} E_{\tilde{\pi}_{1} \tilde{\pi}_{1}}^{1} \pi_{1}+v_{5}^{T} \frac{\sigma_{1}}{\rho_{1}^{2}} E_{\pi_{2} \tilde{\pi}_{1}}^{1} \pi_{1}= \\
\left(v_{4}-v_{3}\right)\left(\nabla_{m_{2}} \kappa_{1}^{1} \frac{\sigma_{1}}{\rho_{1}^{2}} \pi_{1}^{T} \alpha_{1}^{1}-\nabla_{\rho_{1}} \kappa_{1}^{1}\right), \\
v_{2}^{T} \frac{1}{\rho_{1}} E_{\tilde{\pi}_{1} \tilde{\pi}_{1}}^{1} \pi_{1}+v_{5}^{T} \frac{1}{\rho_{1}} E_{\pi_{2} \tilde{\pi}_{1}}^{1} \pi_{1}= \\
\left(v_{4}-v_{3}\right)\left(\nabla_{\sigma_{1}} \kappa_{1}^{1}+\nabla_{m_{2}} \kappa_{1}^{1} \frac{1}{\rho_{1}} \pi_{1}^{T} \alpha_{1}^{1}\right)+\tilde{z}_{3}, \\
v_{5}^{T} \frac{\sigma_{2}}{\rho_{2}^{2}} E_{\tilde{\pi}_{2}}^{2} \tilde{\pi}_{2} \pi_{2}+v_{8}^{T} \frac{\sigma_{2}}{\rho_{2}^{2}} E_{\pi_{3}}^{2} \tilde{\pi}_{2} \pi_{2}= \\
\left(v_{7}-v_{6}\right)\left(\nabla_{m_{3}} \kappa_{2}^{2} \frac{\sigma_{2}}{\rho_{2}^{2}} \alpha_{2}^{2 T} \pi_{2}-\nabla_{\rho_{2}} \kappa_{2}^{2}\right),
\end{gathered}
$$

and

$$
\begin{array}{r}
v_{5}^{T} \frac{1}{\rho_{2}} E_{\tilde{\pi}_{2} \tilde{\pi}_{2}} \pi_{2}+v_{8}^{T} \frac{1}{\rho_{2}} E_{\pi_{3} \tilde{\pi}_{2}}^{2} \pi_{2}= \\
\left(v_{7}-v_{6}\right)\left(\nabla_{\sigma_{2}} \kappa_{2}^{2}+\nabla_{m_{3}} \kappa_{2}^{2} \frac{1}{\rho_{2}} \alpha_{2}^{2 T} \pi_{2}\right)+\tilde{z}_{5},
\end{array}
$$

Post-multiplying (4.46) by the consumer price vector, $\left(\pi^{1 T}, \pi^{2 T}, \pi^{3 T}\right)$ yields

$$
\left(\tilde{z}_{2}, 0_{n-1}^{T}\right) \pi_{1}+\left(\tilde{z}_{4}, 0_{n-1}^{T}\right) \pi_{2}=0
$$

implying that the two multipliers are the consumer prices are not zero and hence that two such normalizations are binding constraints and not consistent with Paretooptimality. Repeating the above exercise with only one consumer price normalisation shows immediately that one price can be normalised. Continuing now as above simply repeats the previous normalisation argument.

We summarize this section with the following result.

Theorem 1: At an efficient optimum with positive savings, one producer price can be normalized in each period, the tax on the initial consumer capital stock can be set equal to zero, either the capital input taxes or the taxes on savings can be set equal to zero in each period (but not both), and at most one consumer tax can be set equal to zero. In addition the government must balance its budget in every period. 
As far as simple normalizations are concerned, this is as far as we can go. If other taxes are to be zero at the optimum, it must result from the structure of the Pareto-set and not merely from some normalisation argument.

\section{The Structure of the Set of Pareto-Optima}

In this section we formally explore the structure of the set of Pareto-optima. More specifically, we show that this set has dimension two. The set of second-best optima are characterized by (3.15); expanding these yields

$$
\begin{gathered}
\xi^{T} P_{\pi}+v^{T}\left[E_{\pi}+E_{p}\right]=0, \\
\xi^{T} P_{\pi}+v^{T} E_{\pi}=0, \\
\xi^{T} P_{m}+v^{T} E_{m}=0, \\
v^{T} E_{\kappa}+\eta^{T}=0
\end{gathered}
$$

and

$$
\theta+\eta^{T} \kappa^{g}=0
$$

Subtracting (5.2) from (5.1) yields

$$
v^{T} E_{p}=0
$$

or, using, (3.8),

$$
\begin{gathered}
v^{1 T}\left[\begin{array}{ccc}
-\nabla_{s_{0}} k_{1} & -\nabla_{p_{1}} k_{1} & -\nabla_{r_{1}} k_{1} \\
\nabla_{s_{0}} a_{1} & \nabla_{p_{1}} a_{1} & \nabla_{r_{1}} a_{1} \\
\nabla_{s_{0}} b_{1} & \nabla_{p_{1}} b_{1} & \nabla_{r_{1}} b_{1}
\end{array}\right]=0, \\
v^{2 T}\left[\begin{array}{ccc}
-\nabla_{s_{1}} k_{2} & -\nabla_{p_{2}} k_{2} & -\nabla_{r_{2}} k_{2} \\
\nabla_{s_{1}} a_{2} & \nabla_{p_{2}} a_{2} & \nabla_{r_{2}} a_{2} \\
\nabla_{s_{1}} b_{2} & \nabla_{p_{2}} b_{2} & \nabla_{r_{2}} b_{2}
\end{array}\right]=0,
\end{gathered}
$$

and

$$
v^{3 T}\left[\begin{array}{cc}
-\nabla_{s_{2}} k_{3} & -\nabla_{p_{3}} k_{3} \\
\nabla_{s_{2}} a_{3} & \nabla_{p_{3}} a_{3}
\end{array}\right]=0
$$


The vector $v$ represents the shadow price of commodity and services to the economy; ${ }^{15}$ these are the prices that should be used to evaluate any public project. Given the strong convexity of the profit functions, the eigenvector associated with the zero eigenvalue of each Hessian in (5.7) is equal to the producer price vector up to a positive multiple. Given the regularity conditions on production (5.7) implies that the social shadow prices are proportional to producer prices so that

$$
\left(v^{1 T}, v^{2 T}, v^{3 T}\right)=\left(\mu_{1}\left[s_{0}, p_{1}^{T}, r_{1}\right], \mu_{2}\left[s_{1}, p_{2}^{T}, r_{2}\right], \mu_{3}\left[s_{2}, p_{3}^{T}\right]\right)
$$

where $\mu_{t}>0$ for $t=1,2,3$. Because of the temporal decomposition of production, $\mu_{t}$ is a function only of the prices in period $t$.

Rewrite (5.1) through (5.6) in conjunction with repeated use of the Slutsky equation to obtain ${ }^{16}$

$$
\begin{gathered}
{\left[\begin{array}{lll}
\mu_{1} p_{1}^{T} & \mu_{2} p_{2}^{T} & \mu_{3} p_{3}^{T}
\end{array}\right]\left[\begin{array}{ccc}
E_{\pi_{1} \pi_{1}}^{0}+\frac{\sigma_{1}}{\rho_{1}} E_{\tilde{\pi}_{1} \tilde{\pi}_{1}}^{1} & E_{\tilde{\pi}_{1} \pi_{2}}^{1} & 0 \\
\frac{\sigma_{1}}{\rho_{1}} E_{\pi_{2} \tilde{\pi}_{1}}^{1} & E_{\pi_{2} \pi_{2}}^{1}+\frac{\sigma_{2}}{\rho_{2}} E_{\tilde{\pi}_{2} \tilde{\pi}_{2}}^{2} & E_{\tilde{\pi}_{2} \pi_{3}}^{2} \\
0 & \frac{\sigma_{2}}{\rho_{2}} E_{\pi_{3} \tilde{\pi}_{2}}^{2} & E_{\pi_{3} \pi_{3}}^{2}
\end{array}\right]=} \\
{\left[\begin{array}{c}
\left(\mu_{2} s_{1}-\mu_{1} r_{1}\right)\left(\nabla_{\pi_{1}} \kappa_{1}^{1}+\nabla_{m_{2}} \kappa_{1}^{1} \frac{\sigma_{1}}{\rho_{1}} \alpha_{1}^{1 T}\right) \\
\left(\mu_{2} s_{1}-\mu_{1} r_{1}\right)\left(\nabla_{\pi_{2}} \kappa_{1}^{1}+\nabla_{m_{2}} \kappa_{1}^{1} \alpha_{2}^{1 T}\right)+\left(\mu_{3} s_{2}-\mu_{2} r_{2}\right)\left(\nabla_{\pi_{2}} \kappa_{2}^{2}+\nabla_{m_{3}} \kappa_{2}^{2} \frac{\sigma_{2}}{\rho_{2}} \alpha_{2}^{2 T}\right) \\
\left(\mu_{3} s_{2}-\mu_{2} r_{2}\right)\left(\nabla_{\pi_{3}} \kappa_{2}^{2}+\nabla_{m_{3}} \kappa_{2}^{2} \alpha_{3}^{2 T}\right)
\end{array}\right]} \\
-\mu_{1} r_{1}+\mu_{2} s_{1}+\eta_{1}=0 \text { and }-\mu_{2} r_{2}+\mu_{3} s_{2}+\eta_{2}=0
\end{gathered}
$$

and the complementary slackness conditions

$$
\eta_{1} \kappa_{1}^{g}=0 \quad \text { and } \quad \eta_{2} \kappa_{2}^{g}=0
$$

In conjunction with (2.14) and (5.8), (5.9) - (5.11) determine the set of Pareto-optima in the economy. First note that there is one more normalisation; dividing (5.9) - (5.11)

15 See Guesnerie $[1977,1995]$ for a detailed argument.

16 See the section entitled Tedious Calculations for details. 
by $\mu_{1}$ does not change the set of optima. Hence there are eight normalizations possible, the seven we established in the previous section plus this one. Next, notice that this set of equations is not linearly independent. Post-multiplying (5.9) by consumer prices, $\left(\pi_{1}^{T}, \pi_{2}^{T}, \pi_{3}^{T}\right)^{T}$, shows that of the $3 n$ equations in (5.9), only $3 n-1$ are linearly independent. Eliminating one of these equations let the mapping determined by (2.14) and (5.9) - (5.10) be called $\phi$; it is a mapping from the space of consumer prices (dimension $3 n+5)$, producer prices (dimension $3 n+5$ ), incomes (dimension 3), and multipliers (dimension $\left.5 ;\left(\mu_{1}, \mu_{2}, \mu_{3}, \eta_{1}, \eta_{2}\right)\right)^{17}$ into an $6 n+8$-dimensional space determined by the $3 n+5$ equilibrium conditions and the additional $3 n+3$ conditions for a Pareto-optimum. Remembering that there are eight normalizations feasible, $\phi: \mathbf{R}^{6 n+10} \mapsto \mathbf{R}^{6 n+8}$. Supposing that $\phi$ is smooth, zero is a regular value of the mapping by construction, thus $\phi^{-1}(0)$ is a smooth manifold of dimension two. ${ }^{18}$ This means that the entire set of Pareto-optima can be parameterized locally in terms of two variables, say, $m_{1}$ and $m_{2}$ or $\tau_{1}^{b}$ and $\tau_{2}^{b}$.

Also of interest is the structure of the tax system in the set of Pareto-optima. That is, for example, are there regions of the Pareto-frontier that entail commodity taxes and taxes on savings and other regions that entail either no taxes or no taxes on savings?

Consider first the case where $\eta_{1}$ and $\eta_{2}$ are equal to zero, that is, neither government capital constraint is strictly binding. This implies that the right side of (5.9) is equal to zero and that

$$
\mu_{1} r_{1}=\mu_{2} s_{1} \quad \text { and } \quad \mu_{2} r_{2}=\mu_{3} s_{2} .
$$

17 The total is $6 n+18$.

18 This follows from the Pre-image Theorem; see Guillemin and Pollack [1974]. In general equilibrium models this is a standard result; see Guesnerie [1979, 1995], and Fuchs and Guesnerie [1983]. If there were $H$ generations instead of three, the Pareto manifold would have dimension $H-1$. 
Rewriting (5.9) now yields

$$
\left[\begin{array}{lll}
p_{1}^{T} & r_{1} p_{2}^{T} & r_{1} r_{2} p_{3}^{T}
\end{array}\right]\left[\begin{array}{ccc}
E_{\pi_{1} \pi_{1}}^{0}+\frac{\sigma_{1}}{\rho_{1}} E_{\tilde{\pi}_{1} \tilde{\pi}_{1}}^{1} & E_{\tilde{\pi}_{1} \pi_{2}}^{1} & 0 \\
\frac{\sigma_{1}}{\rho_{1}} E_{\pi_{2} \tilde{\pi}_{1}}^{1} & E_{\pi_{2} \pi_{2}}^{1}+\frac{\sigma_{2}}{\rho_{2}} E_{\tilde{\pi}_{2} \tilde{\pi}_{2}}^{2} & E_{\tilde{\pi}_{2} \pi_{3}}^{2} \\
0 & \frac{\sigma_{2}}{\rho_{2}} E_{\pi_{3} \tilde{\pi}_{2}}^{2} & E_{\pi_{3} \pi_{3}}^{2}
\end{array}\right]=0 .
$$

First note that in general a set of zero taxes solves this system of equations. Remembering that our normalizations require that $s_{1}=1=s_{2}$, the first element of (5.13) can be written as

$$
p_{1}^{T} E_{\pi_{1} \pi_{1}}^{0}\left(u_{0}, p_{1}\right)+\frac{p_{1}^{T}}{r_{1}} E_{\tilde{\pi}_{1} \tilde{\pi}_{1}}^{1}\left(u_{1}, \frac{p_{1}}{r_{1}}, p_{2}\right)+p_{2}^{T} E_{\pi_{2} \tilde{\pi}_{1}}^{1}\left(u_{1}, \frac{p_{1}}{r_{1}}, p_{2}\right)
$$

which, by homogeneity, is identically zero. Adding (5.14) to the second element of (5.13), factoring out $r_{1}$ and using homogeneity shows that this term is zero. Factoring $r_{1} r_{2}$ out of the third element shows that it too is identically zero by homogeneity. Thus, with positive government and private savings, zero taxes are Pareto-optimal in this region.

Now consider the case where either $\eta_{1}$ or $\eta_{2}$ is not equal to zero; it follows trivially from the above argument that zero taxes cannot be part of the set of Pareto-optima. This is that part of the Pareto-frontier in which the government is trying to redistribute from later generations to earlier ones. Here the non negativity constraints on government capital purchases are binding and the redistribution program must work through the indirect tax system. In this region either taxes on capital inputs or on savings (but not both) are part of the efficient solution.

Theorem 2: The second-best optimum of the overlapping generations model with generation-specific lump-sum taxes is characterized by shadow prices that are proportional to producer prices which, in turn, are proportional to consumer prices only when desired government saving is nonnegative. The set of Pareto-optima has a region of zero taxes and a region of non zero taxes; both regions have dimension two. When desired 
government saving is negative in any period, commodity taxes, taxes on capital inputs or on saving (but not both) are required at almost all optima.

\section{Conclusion}

In this paper we have shown, in a finite-horizon overlapping-generations model, that government intervention in the markets in terms of taxes on commodities and on either savings or capital inputs (but not both) is required for a non negligible set of Paretooptima. This follows basically because the government cannot use the capital market to effect intertemporal transfers of income from later to earlier generations. This imposes a second-best constraint on the model that does not exist in a standard general equilibrium framework.

Our results are of interest not only in their own right, but also in the context of optimal taxes. It is sometimes claimed that expenditure taxes are preferable to income taxes because the latter entail some version of double taxation. ${ }^{19}$ At the very least, it is sometimes maintained that taxes on savings are not optimal. Our results give no support to this claim. At the very least, it is hoped that our results demonstrate that extending the analysis of overlapping generations models to include many consumption goods is both feasible and rewarding.

\section{Tedious Calculations}

This section contains the many tedious calculations necessary for the main results in the previous sections. We first show that the government's budget must be balanced in each period, and then do a complete expansion of the second-best optima before using those calculations to do the expansions for the normalizations.

\footnotetext{
19 See Atkinson and Sandmo [1980], Meade [1975], Ordover and Phelps [1975, 1979], Ordover [1976], and Park [1991].
} 


\subsection{Budget Balance}

It is sufficient to examine period one where government expenditure is given by

$$
m_{1}+r_{1} \kappa_{1}^{g}
$$

Governments revenue, taxes plus profits, is given by

$$
t_{1}^{a T}\left(\alpha_{1}^{0}+\alpha_{1}^{1}\right)+t_{1}^{b} \kappa_{1}^{1}+\left[p_{1}^{T} a_{1}+r_{1} b_{1}-s_{0} \kappa_{0}\right]
$$

Equilibrium requires that

$$
\alpha_{1}^{0}+\alpha_{1}^{1}-a_{1}=0
$$

and

$$
\kappa_{1}^{1}+\kappa_{1}^{g}-b_{1}=0
$$

The consumers are on their respective budget constraints so that

$$
\pi_{1}^{T} \alpha_{1}^{0}=s_{0} \kappa_{0}+m_{1}
$$

and

$$
\pi_{1}^{T} \alpha_{1}^{1}+\rho_{1} \kappa_{1}^{1}=0
$$

Summing the budget constraints and using the definition of consumer prices yields

$$
\left(p_{1}^{T}+t_{1}^{a T}\right)\left(\alpha_{1}^{0}+\alpha_{1}^{1}\right)+r_{1} \kappa_{1}^{1}+t_{1}^{b} \kappa_{1}^{1}=s_{0} \kappa_{0}+m_{1} .
$$

Adding $r_{1} \kappa_{1}^{g}$ to both sides and rearranging yields

$$
\left(p_{1}^{T}+t_{1}^{a T}\right)\left(\alpha_{1}^{0}+\alpha_{1}^{1}\right)+r_{1}\left(\kappa_{1}^{1}+\kappa_{1}^{g}\right)+t_{1}^{b} \kappa_{1}^{1}-s_{0} \kappa_{0}=m_{1}+r_{1} \kappa_{1}^{g} .
$$

Using the equilibrium conditions yields

$$
p_{1}^{T} a_{1}+r_{1} b_{1}-s_{0} \kappa_{0}+t_{1}^{a T}\left(\alpha_{1}^{0}+\alpha_{1}^{1}\right)+t_{1}^{b} \kappa_{1}^{1}=m_{1}+r_{1} \kappa_{1}^{g},
$$

which shows that the government budget is balanced. 


\subsection{Motzkin's Theorem}

We want to show that the non homogeneous system of relations,

$$
A x \gg 0, \quad, B x \geq 0, \quad D x \geq \xi, \quad \text { and } \quad C x=0,
$$

has a solution exactly when there exists a solution to the following homogeneous system:

$$
\left[\begin{array}{cc}
A & 0_{a} \\
0_{n}^{T} & \theta
\end{array}\right]\left[\begin{array}{l}
x \\
z
\end{array}\right] \gg 0, \quad\left[\begin{array}{cc}
B & 0_{b} \\
D & \xi
\end{array}\right]\left[\begin{array}{l}
x \\
z
\end{array}\right] \geq 0, \quad \text { and } \quad\left[\begin{array}{cc}
C & 0_{c} \\
0_{n}^{T} & 0
\end{array}\right]\left[\begin{array}{l}
x \\
z
\end{array}\right]=0
$$

where $x \in \mathcal{R}^{n}$ and $0_{a}$ has the same number of rows as $A$.

If (7.10) has a solution, then (7.11) has a solution with $z=1$. If (7.11) has a solution, then, dividing by through by $z$ shows that (7.10) has a solution.

\subsection{Second-Best Calculations}

We obtain from (5.3)

$$
\xi^{T}+v^{T} E_{m}=0
$$

which becomes

$$
\begin{gathered}
\xi_{1}=\mu_{1} p_{1}^{T} \nabla_{m_{1}} \alpha_{1}^{0} \\
\xi_{2}=\mu_{1} p_{1}^{T} \nabla_{m_{2}} \alpha_{1}^{1}+\left(\mu_{1} r_{1}-\mu_{2} s_{1}\right) \nabla_{m_{2}} \kappa_{1}^{1}+\mu_{2} p_{2}^{T} \nabla_{m_{2}} \alpha_{2}^{1},
\end{gathered}
$$

and

$$
\xi_{3}=\mu_{2} p_{2}^{T} \nabla_{m_{3}} \alpha_{2}^{2}+\left(\mu_{2} r_{2}-\mu_{3} s_{2}\right) \nabla_{m_{3}} \kappa_{2}^{2}+\mu_{3} p_{3}^{T} \nabla_{m_{3}} \alpha_{3}^{2} .
$$

Finally we have from (5.2)

$$
\xi^{T} P_{\pi}+v^{T} E_{\pi}=0
$$

Expanding (7.16) yields

$$
\begin{gathered}
-\xi_{1} \kappa_{0}=-\mu_{1} p_{1}^{T} \nabla_{\sigma_{0}} \alpha_{1}^{0} \\
\xi_{1} \alpha_{1}^{0 T}+\xi_{2} \frac{\sigma_{1}}{\rho_{1}} \alpha_{1}^{1 T}=-\mu_{1} p_{1}^{T} \nabla_{\pi_{1}} \alpha_{1}-\left(\mu_{1} r_{1}-\mu_{2} s_{1}\right) \nabla_{\pi_{1}} \kappa_{1}^{1}-\mu_{2} p_{2}^{T} \nabla_{\pi_{1}} \alpha_{2}^{1}, \\
-\xi_{2} \frac{\sigma_{1}}{\rho_{1}^{2}} \pi_{1}^{T} \alpha_{1}^{1}=-\mu_{1} p_{1}^{T} \nabla_{\rho_{1}} \alpha_{1}^{1}-\left(\mu_{1} r_{1}-\mu_{2} s_{1}\right) \nabla_{\rho_{1}} \kappa_{1}^{1}-\mu_{2} p_{2}^{T} \nabla_{\rho_{1}} \alpha_{2}^{1},
\end{gathered}
$$




$$
\begin{gathered}
\xi_{2} \frac{1}{\rho_{1}} \pi_{1}^{T} \alpha_{1}^{1}=-\mu_{1} p_{1}^{T} \nabla_{\sigma_{1}} \alpha_{1}^{1}-\left(\mu_{1} r_{1}-\mu_{2} s_{1}\right) \nabla_{\sigma_{1}} \kappa_{1}^{1}-\mu_{2} p_{2}^{T} \nabla_{\sigma_{1}} \alpha_{2}^{1} \\
\xi_{2} \alpha_{2}^{1 T}+\xi_{3} \frac{\sigma_{2}}{\rho_{2}} \alpha_{2}^{2 T}=-\mu_{1} p_{1}^{T} \nabla_{\pi_{2}} \alpha_{1}^{1}-\left(\mu_{1} r_{1}-\mu_{2} s_{1}\right) \nabla_{\pi_{2}} \kappa_{1}^{1} \\
\quad-\mu_{2} p_{2}^{T} \nabla_{\pi_{2}} \alpha_{2}-\left(\mu_{2} r_{2}-\mu_{3} s_{2}\right) \nabla_{\pi_{2}} \kappa_{2}^{2}-\mu_{3} p_{3}^{T} \nabla_{\pi_{2}} \alpha_{3}^{2}, \\
-\xi_{3} \frac{\sigma_{2}}{\rho_{2}^{2}} \pi_{2}^{T} \alpha_{2}^{2}=-\mu_{2} p_{2}^{T} \nabla_{\rho_{2}} \alpha_{2}^{2}-\left(\mu_{2} r_{2}-\mu_{3} s_{2}\right) \nabla_{\rho_{2}} \kappa_{2}^{2}-\mu_{3} p_{3}^{T} \nabla_{\rho_{2}} \alpha_{3}^{2}, \\
\xi_{3} \frac{1}{\rho_{2}} \pi_{2}^{T} \alpha_{2}^{2}=-\mu_{2} p_{2}^{T} \nabla_{\sigma_{2}} \alpha_{2}^{2}-\left(\mu_{2} r_{2}-\mu_{3} s_{2}\right) \nabla_{\sigma_{2}} \kappa_{2}^{2}-\mu_{3} p_{3}^{T} \nabla_{\sigma_{2}} \alpha_{3}^{2} \\
\xi_{3} \alpha_{3}^{2 T}=-\mu_{2} p_{2}^{T} \nabla_{\pi_{3}} \alpha_{2}^{2}-\left(\mu_{2} r_{2}-\mu_{3} s_{2}\right) \nabla_{\pi_{3}} \kappa_{2}^{2}-\mu_{3} p_{3}^{T} \nabla_{\pi_{3}} \alpha_{3}^{2} .
\end{gathered}
$$

First note that (7.17) is just (7.13) in disguise. Expanding (7.18)-(7.24), using the values of $\xi_{i}$ from (7.13)-(7.15) and the Slutsky equation repeatedly yields ${ }^{20}$

$$
\begin{aligned}
& \mu_{1} p_{1}^{T} E_{\pi_{1} \pi_{1}}^{0}+\mu_{1} p_{1}^{T} \frac{\sigma_{1}}{\rho_{1}} E_{\tilde{\pi}_{1} \tilde{\pi}_{1}}^{1}+\mu_{2} p_{2}^{T} \frac{\sigma_{1}}{\rho_{1}} E_{\pi_{2} \tilde{\pi}_{1}}^{1}= \\
& \left(\mu_{2} s_{1}-\mu_{1} r_{1}\right)\left(\nabla_{\pi_{1}} \kappa_{1}^{1}+\nabla_{m_{2}} \kappa_{1}^{1} \frac{\sigma_{1}}{\rho_{1}} \alpha_{1}^{1 T}\right) \\
& \mu_{1} p_{1}^{T} \frac{\sigma_{1}}{\rho_{1}^{2}} E_{\tilde{\pi}_{1} \tilde{\pi}_{1}}^{1} \pi_{1}+\mu_{2} p_{2}^{T} \frac{\sigma_{1}}{\rho_{1}^{2}} E_{\pi_{2} \tilde{\pi}_{1}}^{1} \pi_{1}= \\
& \left(\mu_{2} s_{1}-\mu_{1} r_{1}\right)\left(\nabla_{m_{2}} \kappa_{1}^{1} \frac{\sigma_{1}}{\rho_{1}^{2}} \pi_{1}^{T} \alpha_{1}^{1}-\nabla_{\rho_{1}} \kappa_{1}^{1}\right) \\
& \mu_{1} p_{1}^{T} \frac{1}{\rho_{1}} E_{\tilde{\pi}_{1} \tilde{\pi}_{1}}^{1} \pi_{1}+\mu_{2} p_{2}^{T} \frac{1}{\rho_{1}} E_{\pi_{2} \tilde{\pi}_{1}}^{1} \pi_{1}= \\
& \left(\mu_{2} s_{1}-\mu_{1} r_{1}\right)\left(\nabla_{\sigma_{1}} \kappa_{1}^{1}+\nabla_{m_{2}} \kappa_{1}^{1} \frac{1}{\rho_{1}} \pi_{1}^{T} \alpha_{1}^{1}\right) \\
& \mu_{1} p_{1}^{T} E_{\tilde{\pi}_{1} \pi_{2}}^{1}+\mu_{2} p_{2}^{T} E_{\pi_{2} \pi_{2}}^{1}+\mu_{2} p_{2}^{T} \frac{\sigma_{2}}{\rho_{2}} E_{\tilde{\pi}_{2} \tilde{\pi}_{2}}^{2}+\mu_{3} p_{3}^{T} \frac{\sigma_{2}}{\rho_{2}} E_{\pi_{3} \tilde{\pi}_{2}}^{2}= \\
& \left(\mu_{2} s_{1}-\mu_{1} r_{1}\right)\left(\nabla_{\pi_{2}} \kappa_{1}^{1}+\nabla_{m_{2}} \kappa_{1}^{1} \alpha_{2}^{1 T}\right)+\left(\mu_{3} s_{2}-\mu_{2} r_{2}\right)\left(\nabla_{\pi_{2}} \kappa_{2}^{2}+\nabla_{m_{3}} \kappa_{2}^{2} \frac{\sigma_{2}}{\rho_{2}} \alpha_{2}^{2 T}\right), \\
& \mu_{2} p_{2}^{T} \frac{\sigma_{2}}{\rho_{2}^{2}} E_{\tilde{\pi}_{2} \tilde{\pi}_{2}}^{2} \pi_{2}+\mu_{3} p_{3}^{T} \frac{\sigma_{2}}{\rho_{2}^{2}} E_{\pi_{3} \tilde{\pi}_{2}}^{2} \pi_{2}= \\
& \left(\mu_{3} s_{2}-\mu_{2} r_{2}\right)\left(\nabla_{m_{3}} \kappa_{2}^{2} \frac{\sigma_{2}}{\rho_{2}^{2}} \alpha_{2}^{2 T} \pi_{2}-\nabla_{\rho_{2}} \kappa_{2}^{2}\right) \\
& \mu_{2} p_{2}^{T} \frac{1}{\rho_{2}} E_{\tilde{\pi}_{2} \tilde{\pi}_{2}} \pi_{2}+\mu_{3} p_{3}^{T} \frac{1}{\rho_{2}} E_{\pi_{3} \tilde{\pi}_{2}}^{2} \pi_{2}= \\
& \left(\mu_{3} s_{2}-\mu_{2} r_{2}\right)\left(\nabla_{\sigma_{2}} \kappa_{2}^{2}+\nabla_{m_{3}} \kappa_{2}^{2} \frac{1}{\rho_{2}} \alpha_{2}^{2 T} \pi_{2}\right),
\end{aligned}
$$

$\overline{20}$ See the following subsections for the details. 


$$
\begin{aligned}
\mu_{2} p_{2}^{T} E_{\tilde{\pi}_{2} \pi_{3}}^{2}+ & \mu_{3} p_{3}^{T} E_{\pi_{3} \pi_{3}}^{2}= \\
& \left(\mu_{3} s_{2}-\mu_{2} r_{2}\right)\left(\nabla_{\pi_{3}} \kappa_{2}^{2}+\nabla_{m_{3}} \kappa_{2}^{2} \alpha_{3}^{2 T}\right) .
\end{aligned}
$$

Finally, note that writing (7.25), (7.28), and (7.31) in matrix form yields

$$
\begin{gathered}
{\left[\begin{array}{lll}
\mu_{1} p_{1}^{T} & \mu_{2} p_{2}^{T} & \mu_{3} p_{3}^{T}
\end{array}\right]\left[\begin{array}{ccc}
E_{\pi_{1} \pi_{1}}^{0}+\frac{\sigma_{1}}{\rho_{1}} E_{\tilde{\pi}_{1} \tilde{\pi}_{1}}^{1} & E_{\tilde{\pi}_{1} \pi_{2}}^{1} & 0 \\
\frac{\sigma_{1}}{\rho_{1}} E_{\pi_{2} \tilde{\pi}_{1}}^{1} & E_{\pi_{2} \pi_{2}}^{1}+\frac{\sigma_{2}}{\rho_{2}} E_{\tilde{\pi}_{2} \tilde{\pi}_{2}}^{2} & E_{\tilde{\pi}_{2} \pi_{3}}^{2} \\
0 & \frac{\sigma_{2}}{\rho_{2}} E_{\pi_{3} \tilde{\pi}_{2}}^{2} & E_{\pi_{3} \pi_{3}}^{2}
\end{array}\right]=} \\
{\left[\begin{array}{c}
\left(\mu_{2} s_{1}-\mu_{1} r_{1}\right)\left(\nabla_{\pi_{1}} \kappa_{1}^{1}+\nabla_{m_{2}} \kappa_{1}^{1} \frac{\sigma_{1}}{\rho_{1}} \alpha_{1}^{1 T}\right) \\
\left(\mu_{2} s_{1}-\mu_{1} r_{1}\right)\left(\nabla_{\pi_{2}} \kappa_{1}^{1}+\nabla_{m_{2}} \kappa_{1}^{1} \alpha_{2}^{1 T}\right)+\left(\mu_{3} s_{2}-\mu_{2} r_{2}\right)\left(\nabla_{\pi_{2}} \kappa_{2}^{2}+\nabla_{m_{3}} \kappa_{2}^{2} \frac{\sigma_{2}}{\rho_{2}} \alpha_{2}^{2 T}\right)
\end{array}\right],} \\
\left(\mu_{3} s_{2}-\mu_{2} r_{2}\right)\left(\nabla_{\pi_{3}} \kappa_{2}^{2}+\nabla_{m_{3}} \kappa_{2}^{2} \alpha_{3}^{2 T}\right)
\end{gathered}
$$

In addition, the fact that $\kappa_{1}$ is homogeneous of degree zero in $\rho_{1}$ and $\sigma_{1}$ implies that (7.26) and (7.27) are not independent. Similarly, (7.29) and (7.30) are not independent, yielding

$$
\begin{aligned}
\mu_{1} p_{1}^{T} \frac{\sigma_{1}}{\rho_{1}} E_{\tilde{\pi}_{1} \tilde{\pi}_{1}}^{1} \pi_{1}+\mu_{2} p_{2}^{T} \frac{\sigma_{1}}{\rho_{1}} E_{\pi_{2} \tilde{\pi}_{1}}^{1} \pi_{1}= \\
\quad\left(\mu_{2} s_{1}-\mu_{1} r_{1}\right)\left(\nabla_{m_{2}} \kappa_{1}^{1} \frac{\sigma_{1}}{\rho_{1}} \pi_{1}^{T} \alpha_{1}^{1}-\rho_{1} \nabla_{\rho_{1}} \kappa_{1}^{1}\right),
\end{aligned}
$$

and

$$
\begin{array}{r}
\mu_{2} p_{2}^{T} \frac{\sigma_{2}}{\rho_{2}} E_{\tilde{\pi}_{2} \tilde{\pi}_{2}}^{2} \pi_{2}+\mu_{3} p_{3}^{T} \frac{\sigma_{2}}{\rho_{2}} E_{\pi_{3} \tilde{\pi}_{2}}^{2} \pi_{2}= \\
\left(\mu_{3} s_{2}-\mu_{2} r_{2}\right)\left(\nabla_{m_{3}} \kappa_{2}^{2} \frac{\sigma_{2}}{\rho_{2}} \alpha_{2}^{2 T} \pi_{2}-\rho_{2} \nabla_{\rho_{2}} \kappa_{2}^{2}\right) .
\end{array}
$$

Next note that multiplying the first element of (7.32) by $\pi_{1}$ and using the homogeneity of $\kappa_{1}^{1}$ yields(7.33); then multiplying the second element of (7.32) by $\pi_{2}$, adding (7.33) to it and using homogeneity yields (7.34). Finally from (5.4) and (5.5) we have

$$
-\mu_{1} r_{1}+\mu_{2} s_{1}+\eta_{1}=0 \text { and } \quad-\mu_{2} r_{2}+\mu_{3} s_{2}+\eta_{2}=0
$$

and

$$
\theta+\eta_{1} \kappa_{1}^{g}+\eta_{2} \kappa_{2}^{g}=0
$$

Because each element of this is non negative this yields $\theta=0$ and the complementary slackness conditions

$$
\eta_{1} \kappa_{1}^{g}=0 \quad \text { and } \quad \eta_{2} \kappa_{2}^{g}=0
$$


First note that the Slutsky equations can be written as

$$
\begin{gathered}
\nabla_{\pi_{1}} \alpha_{1}^{0}=E_{\pi_{1} \pi_{1}}^{0}-\nabla_{m_{1}} \alpha_{1}^{0} \alpha_{1}^{0 T}, \\
\nabla_{\pi_{1}} \alpha_{1}^{1}=\frac{\sigma_{1}}{\rho_{1}} \nabla_{\tilde{\pi}_{1}} \alpha_{1}^{1}=\frac{\sigma_{1}}{\rho_{1}}\left[E_{\tilde{\pi}_{1} \tilde{\pi}_{1}}^{1}-\nabla_{m_{2}} \alpha_{1}^{1} \alpha_{1}^{1 T}\right], \\
\nabla_{\pi_{1}} \alpha_{2}^{1}=\frac{\sigma_{1}}{\rho_{1}} \nabla_{\tilde{\pi}_{1}} \alpha_{2}^{1}=\frac{\sigma_{1}}{\rho_{1}}\left[E_{\pi_{2} \tilde{\pi}_{1}}^{1}-\nabla_{m_{2}} \alpha_{2}^{1} \alpha_{1}^{1 T}\right] .
\end{gathered}
$$

Substituting these into (7.18) and rearranging yields

$$
\begin{aligned}
& \xi_{1} \alpha_{1}^{0 T}+\xi_{2} \frac{\sigma_{1}}{\rho_{1}} \alpha_{1}^{1 T} \\
& =-\mu_{1} p_{1}^{T} E_{\pi_{1} \pi_{1}}^{0}+\mu_{1} p_{1}^{T} \nabla_{m_{1}} \alpha_{1}^{0} \alpha_{1}^{0 T}-\mu_{1} p_{1}^{T} \frac{\sigma_{1}}{\rho_{1}} E_{\tilde{\pi}_{1} \tilde{\pi}_{1}}^{1}+\mu_{1} p_{1}^{T} \nabla_{m_{2}} \alpha_{1}^{1} \alpha_{1}^{1 T} \\
& \quad-\mu_{2} p_{2}^{T} \frac{\sigma_{1}}{\rho_{1}} E_{\pi_{2} \tilde{\pi}_{1}}^{1}+\mu_{2} p_{2}^{T} \frac{\sigma_{1}}{\rho_{1}} \nabla_{m_{2}} \alpha_{2}^{1} \alpha_{1}^{1 T}-\left(\mu_{1} r_{1}-\mu_{2} s_{1}\right) \nabla_{\pi_{1}} \kappa_{1}^{1} \\
& =-\mu_{1} p_{1}^{T} E_{\pi_{1} \pi_{1}}^{0}-\mu_{1} p_{1}^{T} \frac{\sigma_{1}}{\rho_{1}} E_{\tilde{\pi}_{1} \tilde{\pi}_{1}}^{1}-\mu_{1} p_{1}^{T} \frac{\sigma_{1}}{\rho_{1}} E_{\pi_{2} \tilde{\pi}_{1}}^{1}+\mu_{1} p_{1}^{T} \nabla_{m_{1}} \alpha_{1}^{0} \alpha_{1}^{0 T} \\
& \quad+\frac{\sigma_{1}}{\rho_{1}}\left[\mu_{1} p_{1}^{T} \nabla_{m_{2}} \alpha_{1}^{1}+\mu_{2} p_{2}^{T} \nabla_{m_{2}} \alpha_{2}^{1}\right] \alpha_{1}^{1 T}-\left(\mu_{1} r_{1}-\mu_{2} s_{1}\right) \nabla_{\pi_{1}} \kappa_{1}^{1} \\
& =-\mu_{1} p_{1}^{T} E_{\pi_{1} \pi_{1}}^{0}-\mu_{1} p_{1}^{T} \frac{\sigma_{1}}{\rho_{1}} E_{\tilde{\pi}_{1} \tilde{\pi}_{1}}^{1}-\mu_{1} p_{1}^{T} \frac{\sigma_{1}}{\rho_{1}} E_{\pi_{2} \tilde{\pi}_{1}}^{1} \\
& \quad+\xi_{1} \alpha_{1}^{0 T}+\frac{\sigma_{1}}{\rho_{1}}\left[\xi_{2}-\left(\mu_{1} r_{1}-\mu_{2} s_{1}\right) \nabla_{m_{2}} \kappa_{1}^{1}\right] \alpha_{1}^{1 T}-\left(\mu_{1} r_{1}-\mu_{2} s_{1}\right) \nabla_{\pi_{1}} \kappa_{1}^{1} .
\end{aligned}
$$

Rearranging yields

$$
\begin{aligned}
\mu_{1} p_{1}^{T} E_{\pi_{1} \pi_{1}}^{0} & +\mu_{1} p_{1}^{T} \frac{\sigma_{1}}{\rho_{1}} E_{\tilde{\pi}_{1} \tilde{\pi}_{1}}^{1}+\mu_{1} p_{1}^{T} \frac{\sigma_{1}}{\rho_{1}} E_{\pi_{2} \tilde{\pi}_{1}}^{1} \\
& =\left(\mu_{2} s_{1}-\mu_{1} r_{1}\right)\left[\nabla_{\pi_{1}} \kappa_{1}^{1}+\nabla_{m_{2}} \kappa_{1}^{1}\right] \alpha_{1}^{1 T}
\end{aligned}
$$

which is in fact (7.25). 
Using the Slutsky equations yields

$$
\nabla_{\rho_{1}} \alpha_{1}^{1}=-\frac{\sigma_{1}}{\rho_{1}^{2}} \nabla_{\tilde{\pi}_{1}} \alpha_{1}^{1} \pi_{1}=-\frac{\sigma_{1}}{\rho_{1}^{2}}\left[E_{\tilde{\pi}_{1} \tilde{\pi}_{1}}^{1}-\nabla_{m_{2}} \alpha_{1}^{1} \alpha_{1}^{1 T}\right] \pi_{1}
$$

and

$$
\nabla_{\rho_{1}} \alpha_{2}^{1}=-\frac{\sigma_{1}}{\rho_{1}^{2}} \nabla_{\tilde{\pi}_{1}} \alpha_{2}^{1} \pi_{1}=-\frac{\sigma_{1}}{\rho_{1}^{2}}\left[E_{\pi_{2} \tilde{\pi}_{1}}^{1}-\nabla_{m_{2}} \alpha_{2}^{1} \alpha_{1}^{1 T}\right] \pi_{1} .
$$

Substituting these into (7.19) yields

$$
\begin{gathered}
-\xi_{2} \frac{\sigma_{1}}{\rho_{1}^{2}} \pi_{1}^{T} \alpha_{1}^{1} \\
=\mu_{1} p_{1}^{T} \frac{\sigma_{1}}{\rho_{1}^{2}} E_{\tilde{\pi}_{1} \tilde{\pi}_{1}}^{1} \pi_{1}+\mu_{2} p_{2}^{T} \frac{\sigma_{1}}{\rho_{1}^{2}} E_{\pi_{2} \tilde{\pi}_{1}}^{1} \pi_{1} \\
-\frac{\sigma_{1}}{\rho_{1}^{2}}\left[\mu_{1} p_{1}^{T} \nabla_{m_{2}} \alpha_{1}^{1}+\mu_{2} p_{2}^{T} \nabla_{m_{2}} \alpha_{2}^{1}\right] \alpha_{1}^{1 T} \pi_{1} \\
-\left(\mu_{1} r_{1}-\mu_{2} s_{1}\right) \nabla_{\rho_{1}} \kappa_{1}^{1} \\
=\mu_{1} p_{1}^{T} \frac{\sigma_{1}}{\rho_{1}^{2}} E_{\tilde{\pi}_{1} \tilde{\pi}_{1}}^{1} \pi_{1}+\mu_{2} p_{2}^{T} \frac{\sigma_{1}}{\rho_{1}^{2}} E_{\pi_{2} \tilde{\pi}_{1}}^{1} \pi_{1} \\
-\frac{\sigma_{1}}{\rho_{1}^{2}}\left[\xi_{2}-\left(\mu_{1} r_{1}-\mu_{2} s_{1}\right) \nabla_{m_{2}} \kappa_{1}^{1}\right] \alpha_{1}^{1 T} \pi_{1} \\
-\left(\mu_{1} r_{1}-\mu_{2} s_{1}\right) \nabla_{\rho_{1}} \kappa_{1}^{1} .
\end{gathered}
$$

Rearranging (7.45) yields

$$
\begin{aligned}
\mu_{1} p_{1}^{T} \frac{\sigma_{1}}{\rho_{1}^{2}} E_{\tilde{\pi}_{1}}^{1} \tilde{\pi}_{1} \pi_{1}+\mu_{2} p_{2}^{T} \frac{\sigma_{1}}{\rho_{1}^{2}} E_{\pi_{2} \tilde{\pi}_{1}}^{1} \pi_{1} \\
\quad=\left(\mu_{1} r_{1}-\mu_{2} s_{1}\right)\left[\nabla_{\rho_{1}} \kappa_{1}^{1}-\nabla_{m_{2}} \kappa_{1}^{1} \frac{\sigma_{1}}{\rho_{1}^{2}} \alpha_{1}^{1 T} \pi_{1}\right]
\end{aligned}
$$

which is $(7.26)$.

7.6. (7.20) $\longrightarrow(7.27)$

Using the Slutsky equations yields

$$
\nabla_{\sigma_{1}} \alpha_{1}^{1}=\frac{1}{\rho_{1}} \nabla_{\tilde{\pi}_{1}} \alpha_{1}^{1} \pi_{1}=\frac{1}{\rho_{1}}\left[E_{\tilde{\pi}_{1} \tilde{\pi}_{1}}^{1}-\nabla_{m_{2}} \alpha_{1}^{1} \alpha_{1}^{1 T}\right] \pi_{1}
$$


and

$$
\nabla_{\sigma_{1}} \alpha_{2}^{1}=\frac{1}{\rho_{1}} \nabla_{\tilde{\pi}_{1}} \alpha_{2}^{1} \pi_{1}=\frac{1}{\rho_{1}}\left[E_{\pi_{2} \tilde{\pi}_{1}}^{1}-\nabla_{m_{2}} \alpha_{2}^{1} \alpha_{1}^{1 T}\right] \pi_{1} .
$$

Substituting these into (7.19) yields

$$
\begin{aligned}
& \xi_{2} \frac{1}{\rho_{1}} \alpha_{1}^{1 T} \pi_{1} \\
&=-\frac{1}{\rho_{1}}\left[\mu_{1} p_{1}^{T} E_{\tilde{\pi}_{1} \tilde{\pi}_{1}}^{1}+\mu_{2} p_{2}^{T} E_{\pi_{2} \tilde{\pi}_{1}}^{1}\right] \pi_{1} \\
& \quad+\frac{1}{\rho_{1}}\left[\mu_{1} p_{1}^{T} \nabla_{m_{2}} \alpha_{1}^{1}+\mu_{2} p_{2}^{T} \nabla_{m_{2}} \alpha_{2}^{1}\right] \alpha_{1}^{1 T} \pi_{1} \\
& \quad-\left(\mu_{1} r_{1}-\mu_{2} s_{1}\right) \nabla_{\sigma_{1}} \kappa_{1}^{1} \\
&=-\frac{1}{\rho_{1}}\left[\mu_{1} p_{1}^{T} E_{\tilde{\pi}_{1}}^{1} \tilde{\pi}_{1}+\mu_{2} p_{2}^{T} E_{\pi_{2}}^{1} \tilde{\pi}_{1}\right] \pi_{1} \\
& \frac{1}{\rho_{1}}\left[\xi_{2}-\left(\mu_{1} r_{1}-\mu_{2} s_{1}\right) \nabla_{m_{2}} \kappa_{1}^{1}\right] \alpha_{1}^{1 T} \pi_{1} \\
& \quad-\left(\mu_{1} r_{1}-\mu_{2} s_{1}\right) \nabla_{\sigma_{1}} \kappa_{1}^{1} .
\end{aligned}
$$

Rearranging yields

$$
\begin{aligned}
& \frac{1}{\rho_{1}}\left[\mu_{1} p_{1}^{T} E_{\tilde{\pi}_{1} \tilde{\pi}_{1}}^{1}+\mu_{2} p_{2}^{T} E_{\pi_{2} \tilde{\pi}_{1}}^{1}\right] \pi_{1} \\
& \quad=\left(\mu_{2} s_{1}-\mu_{1} r_{1}\right)\left[\nabla_{\sigma_{1}} \kappa_{1}^{1}+\frac{1}{\rho_{1}} \nabla_{m_{2}} \kappa_{1}^{1} \alpha_{1}^{1 T} \pi_{1}\right]
\end{aligned}
$$

which is (7.27).

7.7. (7.21) $\longrightarrow(7.28)$

Using the Slutsky equations yields

$$
\begin{gathered}
\nabla_{\pi_{2}} \alpha_{1}^{1}=E_{\pi_{2} \pi_{2}}^{1}-\nabla_{m_{2}} \alpha_{1}^{1} \alpha_{2}^{1 T}, \\
\nabla_{\pi_{2}} \alpha_{2}^{1}=E_{\tilde{\pi}_{1} \pi_{2}}^{1}-\nabla_{m_{2}} \alpha_{1}^{1} \alpha_{2}^{1 T}, \\
\nabla_{\pi_{2}} \alpha_{2}^{2}=\frac{\sigma_{2}}{\rho_{2}} \nabla_{\tilde{\pi}_{2}} \alpha_{2}^{2}=\frac{\sigma_{2}}{\rho_{2}}\left[E_{\tilde{\pi}_{2} \tilde{\pi}_{2}}^{2}-\nabla_{m_{3}} \alpha_{2}^{2} \alpha_{2}^{2 T}\right],
\end{gathered}
$$

and

$$
\nabla_{\pi_{2}} \alpha_{3}^{2}=\frac{\sigma_{2}}{\rho_{2}}\left[E_{\pi_{3} \tilde{\pi}_{2}}^{2}-\nabla_{m_{3}} \alpha_{3}^{2} \alpha_{2}^{2 T}\right]
$$


Substituting these into (7.21) yields

$$
\begin{aligned}
\xi_{2} \alpha_{2}^{1 T}+ & \xi_{3} \frac{\sigma_{2}}{\rho_{2}} \alpha_{2}^{2 T} \\
=- & \mu_{1} p_{1}^{T} E_{\tilde{\pi}_{1} \pi_{2}}^{1}-\mu_{2} p_{2}^{T} E_{\pi_{2} \pi_{2}}^{1}-\mu_{2} p_{2}^{T} \frac{\sigma_{2}}{\rho_{2}} E_{\tilde{\pi}_{2} \tilde{\pi}_{2}}^{2}-\mu_{3} p_{3}^{T} \frac{\sigma_{2}}{\rho_{2}} E_{\pi_{3} \tilde{\pi}_{2}}^{2} \\
& +\left[\mu_{1} p_{1}^{T} \nabla_{m_{2}} \alpha_{1}^{1}+\mu_{2} p_{2}^{T} \nabla_{m_{2}} \alpha_{2}^{1}\right]+\frac{\sigma_{2}}{\rho_{2}}\left[\mu_{2} p_{2}^{T} \nabla_{m_{3}} \alpha_{2}^{2}+\mu_{3} p_{3}^{T} \nabla_{m_{3}} \alpha_{3}^{2}\right] \\
& -\left(\mu_{1} r_{1}-\mu_{2} s_{1}\right) \nabla_{\pi_{2}} \kappa_{1}^{1}-\left(\mu_{2} r_{2}-\mu_{3} s_{2}\right) \nabla_{\pi_{2}} \kappa_{2}^{2} \\
=- & \mu_{1} p_{1}^{T} E_{\tilde{\pi}_{1} \pi_{2}}^{1}-\mu_{2} p_{2}^{T} E_{\pi_{2} \pi_{2}}^{1}-\mu_{2} p_{2}^{T} \frac{\sigma_{2}}{\rho_{2}} E_{\tilde{\pi}_{2} \tilde{\pi}_{2}}^{2}-\mu_{3} p_{3}^{T} \frac{\sigma_{2}}{\rho_{2}} E_{\pi_{3} \tilde{\pi}_{2}}^{2} \\
& +\left[\xi_{2}-\left(\mu_{1} r_{1}-\mu_{2} s_{1}\right) \nabla_{m_{2}} \kappa_{1}^{1}\right] \alpha_{2}^{1 T}+\frac{\sigma_{2}}{\rho_{2}}\left[\xi_{3}-\left(\mu_{2} r_{2}-\mu_{3} s_{2}\right) \nabla_{m_{3}} \kappa_{2}^{2}\right] \alpha_{2}^{2 T} \\
& -\left(\mu_{1} r_{1}-\mu_{2} s_{1}\right) \nabla_{\pi_{2}} \kappa_{1}^{1}-\left(\mu_{2} r_{2}-\mu_{3} s_{2}\right) \nabla_{\pi_{2}} \kappa_{2}^{2} .
\end{aligned}
$$

Rearranging (7.55) yields

$$
\begin{aligned}
& \mu_{1} p_{1}^{T} E_{\tilde{\pi}_{1} \pi_{2}}^{1}+\mu_{2} p_{2}^{T} E_{\pi_{2} \pi_{2}}^{1}+\mu_{2} p_{2}^{T} \frac{\sigma_{2}}{\rho_{2}} E_{\tilde{\pi}_{2} \tilde{\pi}_{2}}^{2}+\mu_{3} p_{3}^{T} \frac{\sigma_{2}}{\rho_{2}} E_{\pi_{3} \tilde{\pi}_{2}}^{2} \\
&=\left(\mu_{2} s_{1}-\mu_{1} r_{1}\right)\left[\nabla_{\pi_{2}} \kappa_{1}^{1}+\nabla_{m_{2}} \kappa_{1}^{1} \alpha_{2}^{1 T}\right] \\
& \quad+\left(\mu_{3} s_{2}-\mu_{2} r_{2}\right)\left[\nabla_{\pi_{2}} \kappa_{2}^{2}+\frac{\sigma_{2}}{\rho_{2}} \nabla_{m_{3}} \kappa_{2}^{2} \alpha_{2}^{2 T}\right]
\end{aligned}
$$

which is (7.28).

7.8. (7.22) $\longrightarrow(7.29)$

The Slutsky equations yield

$$
\nabla_{\rho_{2}} \alpha_{2}^{2}=\frac{\sigma_{2}}{\rho_{2}^{2}} \nabla_{\tilde{\pi}_{2}} \alpha_{2}^{2} \pi_{2}^{T}=-\frac{\sigma_{2}}{\rho_{2}^{2}}\left[E_{\tilde{\pi}_{2}}^{2} \tilde{\pi}_{2}-\nabla_{m_{3}} \alpha_{2}^{2} \alpha_{2}^{2 T}\right] \pi_{2}
$$

and

$$
\nabla_{\rho_{2}} \alpha_{3}^{2}=-\frac{\sigma_{2}}{\rho_{2}^{2}}\left[E_{\pi_{3}}^{2} \tilde{\pi}_{2}-\nabla_{m_{3}} \alpha_{3}^{2} \alpha_{2}^{2 T}\right] \pi_{2}
$$


Substituting these into (7.22) yields

$$
\begin{aligned}
-\xi_{3} \frac{\sigma_{2}}{\rho_{2}^{2}} \alpha_{2}^{2 T} \pi_{2} & \\
=\mu_{2} p_{2}^{T} & \frac{\sigma_{2}}{\rho_{2}^{2}} E_{\tilde{\pi}_{2} \tilde{\pi}_{2}}^{2} \pi_{2}+\mu_{3} p_{3}^{T} \frac{\sigma_{2}}{\rho_{2}^{2}} E_{\pi_{3}}^{2} \tilde{\pi}_{2} \pi_{2} \\
& \quad-\frac{\sigma_{2}}{\rho_{2}^{2}}\left[\mu_{2} p_{2}^{T} \nabla_{m_{3}} \alpha_{2}^{2}+\mu_{3} p_{3}^{T} \nabla_{m_{3}} \alpha_{3}^{2}\right] \alpha_{2}^{2 T} \pi_{2} \\
& -\left(\mu_{2} r_{2}-\mu_{3} s_{2}\right) \nabla_{\rho_{2}} \kappa_{2}^{2} \\
=\mu_{2} p_{2}^{T} & \frac{\sigma_{2}}{\rho_{2}^{2}} E_{\tilde{\pi}_{2}}^{2} \tilde{\pi}_{2} \pi_{2}+\mu_{3} p_{3}^{T} \frac{\sigma_{2}}{\rho_{2}^{2}} E_{\pi_{3}}^{2} \tilde{\pi}_{2} \pi_{2} \\
& \quad-\frac{\sigma_{2}}{\rho_{2}^{2}}\left[\xi_{3}-\left(\mu_{2} r_{2}-\mu_{3} s_{2}\right) \nabla_{m_{3}} \kappa_{2}^{2}\right] \alpha_{2}^{2 T} \pi_{2} \\
& \quad-\left(\mu_{2} r_{2}-\mu_{3} s_{2}\right) \nabla_{\rho_{2}} \kappa_{2}^{2}
\end{aligned}
$$

Rearranging (4.26) yields

$$
\begin{aligned}
\mu_{2} p_{2}^{T} & \frac{\sigma_{2}}{\rho_{2}^{2}} E_{\tilde{\pi}_{2}}^{2} \tilde{\pi}_{2} \pi_{2}+\mu_{3} p_{3}^{T} \frac{\sigma_{2}}{\rho_{2}^{2}} E_{\pi_{3} \tilde{\pi}_{2}}^{2} \pi_{2} \\
& =\left(\mu_{3} s_{2}-\mu_{2} r_{2}\right)\left[\frac{\sigma_{2}}{\rho_{2}^{2}} \nabla_{m_{3}} \kappa_{2}^{2} \alpha_{2}^{2 T} \pi_{2}-\nabla_{\rho_{2}} \kappa_{2}^{2}\right]
\end{aligned}
$$

which is (7.29).

\subsection{Normalisation Calculations}

Collecting (4.29) - (4.34) of the text and using the Slutsky equation yields

$$
\begin{gathered}
-\xi_{1} \kappa_{0}=-v_{2}^{T} \nabla_{\sigma_{0}} \alpha_{1}^{0}+z_{1} \\
\xi_{1} \alpha_{1}^{0 T}+\xi_{2} \frac{\sigma_{1}}{\rho_{1}} \alpha_{1}^{1 T}=-v_{2}^{T} \nabla_{\pi_{1}} \alpha_{1}-\left(v_{3}-v_{4}\right) \nabla_{\pi_{1}} \kappa_{1}^{1}-v_{5}^{T} \nabla_{\pi_{1}} \alpha_{2}^{1} \\
-\xi_{2} \frac{\sigma_{1}}{\rho_{1}^{2}} \pi_{1}^{T} \alpha_{1}^{1}=-v_{2}^{T} \nabla_{\rho_{1}} \alpha_{1}^{1}-\left(v_{3}-v_{4}\right) \nabla_{\rho_{1}} \kappa_{1}^{1}-v_{5}^{T} \nabla_{\rho_{1}} \alpha_{2}^{1} \\
\xi_{2} \frac{1}{\rho_{1}} \pi_{1}^{T} \alpha_{1}^{1}=-v_{2}^{T} \nabla_{\sigma_{1}} \alpha_{1}^{1}-\left(v_{3}-v_{4}\right) \nabla_{\sigma_{1}} \kappa_{1}^{1}-v_{5}^{T} \nabla_{\sigma_{1}} \alpha_{2}^{1}+z_{2}, \\
\xi_{2} \alpha_{2}^{1 T}+\xi_{3} \frac{\sigma_{2}}{\rho_{2}} \alpha_{2}^{2 T}=-v_{2}^{T} \nabla_{\pi_{2}} \alpha_{1}^{1}-\left(v_{3}-v_{4}\right) \nabla_{\pi_{2}} \kappa_{1}^{1} \\
\quad-v_{5}^{T} \nabla_{\pi_{2}} \alpha_{2}-\left(v_{6}-v_{7}\right) \nabla_{\pi_{2}} \kappa_{2}^{2}-v_{8}^{T} \nabla_{\pi_{2}} \alpha_{3}^{2}, \\
-\xi_{3} \frac{\sigma_{2}}{\rho_{2}^{2}} \pi_{2}^{T} \alpha_{2}^{2}=-v_{5}^{T} \nabla_{\rho_{2}} \alpha_{2}^{2}-\left(v_{6}-v_{7}\right) \nabla_{\rho_{2}} \kappa_{2}^{2}-v_{8}^{T} \nabla_{\rho_{2}} \alpha_{3}^{2}
\end{gathered}
$$




$$
\begin{aligned}
\xi_{3} \frac{1}{\rho_{2}} \pi_{2}^{T} \alpha_{2}^{2} & =-v_{5}^{T} \nabla_{\sigma_{2}} \alpha_{2}^{2}-\left(v_{6}-v_{7}\right) \nabla_{\sigma_{2}} \kappa_{2}^{2}-v_{8}^{T} \nabla_{\sigma_{2}} \alpha_{3}^{2}+z_{3} \\
\xi_{3} \alpha_{3}^{2 T} & =-v_{5}^{T} \nabla_{\pi_{3}} \alpha_{2}^{2}-\left(v_{6}-v_{7}\right) \nabla_{\pi_{3}} \kappa_{2}^{2}-v_{8}^{T} \nabla_{\pi_{3}} \alpha_{3}^{2}
\end{aligned}
$$

Expanding (7.62)-(7.68), using the values of $\xi_{i}$ from (4.26)-(4.28) and the Slutsky equation repeatedly yields

$$
\begin{aligned}
& v_{2}^{T} E_{\pi_{1} \pi_{1}}^{0}+v_{2}^{T} \frac{\sigma_{1}}{\rho_{1}} E_{\tilde{\pi}_{1} \tilde{\pi}_{1}}^{1}+v_{5}^{T} \frac{\sigma_{1}}{\rho_{1}} E_{\pi_{2} \tilde{\pi}_{1}}^{1}= \\
& \left(v_{4}-v_{3}\right)\left(\nabla_{\pi_{1}} \kappa_{1}^{1}+\nabla_{m_{2}} \kappa_{1}^{1} \frac{\sigma_{1}}{\rho_{1}} \alpha_{1}^{1 T}\right), \\
& v_{2}^{T} \frac{\sigma_{1}}{\rho_{1}^{2}} E_{\tilde{\pi}_{1} \tilde{\pi}_{1}}^{1} \pi_{1}+v_{5}^{T} \frac{\sigma_{1}}{\rho_{1}^{2}} E_{\pi_{2} \tilde{\pi}_{1}}^{1} \pi_{1}= \\
& \left(v_{4}-v_{3}\right)\left(\nabla_{m_{2}} \kappa_{1}^{1} \frac{\sigma_{1}}{\rho_{1}^{2}} \pi_{1}^{T} \alpha_{1}^{1}-\nabla_{\rho_{1}} \kappa_{1}^{1}\right), \\
& v_{2}^{T} \frac{1}{\rho_{1}} E_{\tilde{\pi}_{1} \tilde{\pi}_{1}}^{1} \pi_{1}+v_{5}^{T} \frac{1}{\rho_{1}} E_{\pi_{2} \tilde{\pi}_{1}}^{1} \pi_{1}= \\
& \left(v_{4}-v_{3}\right)\left(\nabla_{\sigma_{1}} \kappa_{1}^{1}+\nabla_{m_{2}} \kappa_{1}^{1} \frac{1}{\rho_{1}} \pi_{1}^{T} \alpha_{1}^{1}\right)+z_{2}, \\
& v_{2}^{T} E_{\tilde{\pi}_{1} \pi_{2}}^{1}+v_{5}^{T} E_{\pi_{2} \pi_{2}}^{1}+v_{5}^{T} \frac{\sigma_{2}}{\rho_{2}} E_{\tilde{\pi}_{2}}^{2} \tilde{\pi}_{2}+v_{8}^{T} \frac{\sigma_{2}}{\rho_{2}} E_{\pi_{3}}^{2} \tilde{\pi}_{2}= \\
& \left(v_{4}-v_{3}\right)\left(\nabla_{\pi_{2}} \kappa_{1}^{1}+\nabla_{m_{2}} \kappa_{1}^{1} \alpha_{2}^{1 T}\right)+\left(v_{7}-v_{6}\right)\left(\nabla_{\pi_{2}} \kappa_{2}^{2}+\nabla_{m_{3}} \kappa_{2}^{2} \frac{\sigma_{2}}{\rho_{2}} \alpha_{2}^{2 T}\right) \text {, } \\
& v_{5}^{T} \frac{\sigma_{2}}{\rho_{2}^{2}} E_{\tilde{\pi}_{2} \tilde{\pi}_{2}}^{2} \pi_{2}+v_{8}^{T} \frac{\sigma_{2}}{\rho_{2}^{2}} E_{\pi_{3} \tilde{\pi}_{2}}^{2} \pi_{2}= \\
& \left(v_{7}-v_{6}\right)\left(\nabla_{m_{3}} \kappa_{2}^{2} \frac{\sigma_{2}}{\rho_{2}^{2}} \alpha_{2}^{2 T} \pi_{2}-\nabla_{\rho_{2}} \kappa_{2}^{2}\right), \\
& v_{5}^{T} \frac{1}{\rho_{2}} E_{\tilde{\pi}_{2} \tilde{\pi}_{2}} \pi_{2}+v_{8}^{T} \frac{1}{\rho_{2}} E_{\pi_{3} \tilde{\pi}_{2}}^{2} \pi_{2}= \\
& \left(v_{7}-v_{6}\right)\left(\nabla_{\sigma_{2}} \kappa_{2}^{2}+\nabla_{m_{3}} \kappa_{2}^{2} \frac{1}{\rho_{2}} \alpha_{2}^{2 T} \pi_{2}\right)+z_{3} \text {, } \\
& v_{5}^{T} E_{\tilde{\pi}_{2} \pi_{3}}^{2}+v_{8}^{T} E_{\pi_{3} \pi_{3}}^{2}= \\
& \left(v_{7}-v_{6}\right)\left(\nabla_{\pi_{3}} \kappa_{2}^{2}+\nabla_{m_{3}} \kappa_{2}^{2} \alpha_{3}^{2 T}\right) .
\end{aligned}
$$

Rewrite (7.69), (7.72), and (7.75) in matrix form to obtain 


$$
\begin{gathered}
{\left[\begin{array}{lll}
v_{2}^{T} & v_{5}^{T} & v_{8}^{T}
\end{array}\right]\left[\begin{array}{ccc}
E_{\pi_{1} \pi_{1}}^{0}+\frac{\sigma_{1}}{\rho_{1}} E_{\tilde{\pi}_{1} \tilde{\pi}_{1}}^{1} & E_{\tilde{\pi}_{1} \pi_{2}}^{1} & 0 \\
\frac{\sigma_{1}}{\rho_{1}} E_{\pi_{2} \tilde{\pi}_{1}}^{1} & E_{\pi_{2} \pi_{2}}^{1}+\frac{\sigma_{2}}{\rho_{2}} E_{\tilde{\pi}_{2} \tilde{\pi}_{2}}^{2} & E_{\tilde{\pi}_{2} \pi_{3}}^{2} \\
0 & \frac{\sigma_{2}}{\rho_{2}} E_{\pi_{3}}^{2} \tilde{\pi}_{2} & E_{\pi_{3} \pi_{3}}^{2}
\end{array}\right]=} \\
{\left[\begin{array}{c}
\left(v_{4}-v_{3}\right)\left(\nabla_{\pi_{1}} \kappa_{1}^{1}+\nabla_{m_{2}} \kappa_{1}^{1} \frac{\sigma_{1}}{\rho_{1}} \alpha_{1}^{1 T}\right) \\
\left(v_{4}-v_{3}\right)\left(\nabla_{\pi_{2}} \kappa_{1}^{1}+\nabla_{m_{2}} \kappa_{1}^{1} \alpha_{2}^{1 T}\right)+\left(v_{7}-v_{6}\right)\left(\nabla_{\pi_{2}} \kappa_{2}^{2}+\nabla_{m_{3}} \kappa_{2}^{2} \frac{\sigma_{2}}{\rho_{2}} \alpha_{2}^{2 T}\right) \\
\left(v_{7}-v_{6}\right)\left(\nabla_{\pi_{3}} \kappa_{2}^{2}+\nabla_{m_{3}} \kappa_{2}^{2} \alpha_{3}^{2 T}\right)
\end{array}\right] .}
\end{gathered}
$$

\section{REFERENCES}

Allais, M., Economie et Intérêt, Imprimerie Nationale, Paris, 1947.

Atkinson, A. and A. Sandmo, "Welfare Implications of the Taxation of Savings", Economic Journal 90, 1980, 529-549.

Balasko, Y. and K. Shell, "The Overlapping Generations Model, I: the Case of Pure Exchange Without Money", Journal of Economic Theory 23, 1980, 281-306.

Balasko, Y. and K. Shell, "The Overlapping Generations Model, I: the Case of Pure Exchange With Money", Journal of Economic Theory 24, 1981, 112-142.

Balasko, Y. and K. Shell, "Lump-Sum Taxes and Transfers: Public Debt in the Overlapping Generations Model" in Equilibrium Analysis: Essays in Honor of Kenneth J. Arrow, V. II, edited by W. Heller Jr., R. Starr and D. Starret, 1986.

Blackorby, C. and C. Brett, "Fiscal Federalism Revisited", UBC Discussion Paper 98-09, 1998 (http://web.arts.ubc.ca/econ/), forthcoming in Journal of Economic Theory.

Blackorby, C. and W. Diewert, "Expenditure Functions, Local Duality, and Second Order Approximations", Econometrica 47, 1979, 579-601.

Diamond, P., "National Debt in a Neoclassical Growth Model", American Economic Review 55, 1965, 1125-1150.

Diewert, W., "Optimal Tax Perturbations", Journal of Public Economics 10, 1978, 139-177.

Diewert, W., M. Avriel, and I. Zang, "Nine Kinds of Quasi-Concavity and Concavity", Journal of Economic Theory 25, 1981, 397-420.

Fuchs, G. and R. Guesnerie, "Structure of Tax Equilibria", Econometrica 51, 1983, 403-434.

Ghiglino, C. and K. Shell, "The Economic Effects of Restrictions on Government Budget Deficits", mimeo, copenhagen Business School and Cornell University. 
Guilemin, V. and A. Pollack, Differential Topology, Prentice-Hall, 1974.

Guesnerie, R., "On The Direction of Tax Reform", Journal of Public Economics 7, 1977, 179-202.

Guesnerie, R., "Financing Public Goods with Taxes: The Tax Reform View Point", Econometrica 47, 1979, 393-421.

Guesnerie, R., A Contribution to the Pure Theory of Taxation, Cambridge University Press, 1995.

Mangasarian, O., Nonlinear Programming, McGraw-Hill, 1969.

Meade, J., The Intelligent Radical's Guide to Economic Policy, Allen and Unwin, 1975.

Myles, G., Public Economics, Cambridge University Press, 1995.

Ordover, J. "Distributive Justice and Optimal Taxation of Wages and Interest in a Growing Economy", Journal of Public Economics 5, 1976, 139-160.

Ordover, J. and E. Phelps, "Linear Taxation of Wealth and Wages for Intragenerational Lifetime Justice: Some Steady-State Cases", American Economic Review 65, 1975, 660-673.

Ordover, J. and E. Phelps, "The Concept of of Optimal Taxation in the OverlappingGenerations Model of Capital and Wealth", Journal of Public Economics 12, 1979, $1-26$.

Park, N. "Steady-State Solutions of Optimal Tax Mixes in and Overlapping-Generations Model", Journal of Public Economics 46, 1991, 227-246.

Samuelson, P., "An Exact Consumption-Loan Model of Interest With or Without the Social Contrivance of Money, Journal of Political Economy 46, 1958, 467-482.

Weymark, J., "A Reconciliation of Recent Results in Optimal Taxation", Journal of Public Economics 12, 1979,171-189. 